\title{
On nilpotent operators
}

\author{
by \\ Laura Burlando (Genova)
}

\begin{abstract}
We give several necessary and sufficient conditions in order that a bounded linear operator on a Banach space be nilpotent. We also discuss three necessary conditions for nilpotency. Furthermore, we construct an infinite family (in one-to-one correspondence with the square-summable sequences $\left(\varepsilon_{n}\right)_{n \in \mathbb{N}}$ of strictly positive real numbers) of nonnilpotent quasinilpotent operators on an infinite-dimensional Hilbert space, all the iterates of each of which have closed range. Each of these operators (as well as an operator previously constructed by C. Apostol in [Ap]) can be used to provide a negative answer to a question posed by M. Mbekhta and J. Zemánek [MZ]. We also use our example to show that two (equivalent to each other) of the three necessary conditions for nilpotency we have mentioned above are not sufficient, by proving that the sequence $\left(\varepsilon_{n}\right)_{n \in \mathbb{N}}$ can be chosen so that these two conditions are satisfied. Finally, from a generalization-obtained by using a theorem proved by M. Gonzalez and V. M. Onieva in [GO2] - of a result provided by $\mathrm{C}$. Apostol in [Ap], we derive that any holomorphic function of each operator in our example, as well as of the one constructed in [Ap], has closed range.
\end{abstract}

1. Introduction. Throughout this paper, when the scalar field is not specified, it may be either $\mathbb{C}$ or $\mathbb{R}$.

For each vector space $V$, let $0_{V}$ and $I_{V}$ denote respectively the zero element of $V$ and the identity operator on $V$. If $E$ is an endomorphism of $V$, we denote the kernel and the range of $E$ by $\mathcal{N}(E)$ and $\mathcal{R}(E)$, respectively. If $X$ is a Banach space, $L(X)$ stands for the Banach algebra of all bounded linear operators on $X$. If $T \in L(X)$ is invertible in $L(X)$, we denote the inverse of $T$ in $L(X)$ by $T^{-1}$.

If $\mathcal{X}$ is a complex Banach space and $A \in L(\mathcal{X})$, let $\sigma(A)$ stand for the spectrum of $A$. It is well known that the resolvent function of $A$

$$
R(\cdot, A): \mathbb{C} \backslash \sigma(A) \ni \lambda \mapsto\left(\lambda I_{\mathcal{X}}-A\right)^{-1} \in L(\mathcal{X})
$$

is holomorphic on $\mathbb{C} \backslash \sigma(A)$.

2000 Mathematics Subject Classification: Primary 47A05.

Key words and phrases: nilpotent and quasinilpotent operators, closed ranges of the iterates, ascent, reduced minimum modulus. 
For every normed space $Y$, we denote the norm of $Y$ by \|\|$_{Y}$. Also, let $\mathbb{N}$ and $\mathbb{Z}_{+}$stand for the sets of all nonnegative integers and of all positive integers, respectively.

We recall that a bounded linear operator $T$ on a Banach space $X$ is called:

- nilpotent if there exists $p \in \mathbb{N}$ such that $T^{p}=0_{L(X)}$;

- quasinilpotent if $\lim _{n \rightarrow \infty} \sqrt[n]{\left\|T^{n}\right\|_{L(X)}}=0$.

Clearly, every nilpotent operator is quasinilpotent. Now suppose the Banach space $X$ is nonzero and let $T \in L(X)$. Since $T^{0}=I_{X} \neq 0_{L(X)}$, if $T$ is nilpotent we have

$$
\min \left\{p \in \mathbb{N}: T^{p}=0_{L(X)}\right\} \in \mathbb{Z}_{+} .
$$

We also recall that, if $X$ is complex, then $T$ is quasinilpotent if and only if $\sigma(T)=\{0\}$ (see [TL, $\mathrm{V}, 3.5]$ ); moreover, $T$ is nilpotent if and only if $\sigma(T)=\{0\}$ and 0 is a pole of $R(\cdot, T)$ (see [TL, V, 10.5 and 10.6]). In the real case, $T$ being quasinilpotent is equivalent to the spectrum of the complex extension $\widetilde{T}$ of $T$ to the complexification $\widetilde{X}$ of $X$ (see [Sc, p. 261]) being equal to $\{0\}$ (as it is not difficult to verify that $\left\|(\widetilde{T})^{n}\right\|_{L(\widetilde{X})}=\left\|T^{n}\right\|_{L(X)}$ for all $n \in \mathbb{N}$ ), and $T$ being nilpotent is equivalent to $\sigma(\widetilde{T})=\{0\}$ plus 0 being a pole of $R(\cdot, \widetilde{T})$.

The problem of finding conditions ensuring that a bounded linear operator $T$ on a Banach space $X$ be nilpotent (or the equivalent one of finding conditions ensuring that $T-I_{X}$ be nilpotent) has been considered by several authors (see also [Ze] for an extensive bibliography about this topic). Extending I. Gelfand's characterization of the identity operator among all the ones having spectrum equal to $\{1\}$ ([Ge2, Theorem 1]), E. Hille ([H, p. 59]; see also [St, p. 6], [HP, Theorem 4.10.1] and [Ze, Theorem 2]) characterized nilpotency of $T-I_{X}$ (where $X$ is a complex Banach space), under the hypothesis $\sigma(T)=\{1\}$, in terms of the asymptotic behaviour of the sequence $\left(T^{n}\right)_{n \in \mathbb{Z}}$ as $|n| \rightarrow \infty$. Further necessary and sufficient conditions for nilpotency of $T-I_{X}$, for $T$ satisfying $\sigma(T)=\{1\}$, in terms of the asymptotic behaviour of the sequence above as $|n| \rightarrow \infty$, were more recently obtained by A. Atzmon [At], J. Zemánek [Ze], M. Zarrabi [Za], and D. Drissi and J. Zemánek [DZ]: indeed, a characterization of this kind follows from Corollary 1 of [At], another one can be derived from Theorem 4 of [Ze] and further ones are stated in $[\mathrm{Za}, 5.1]$ and in [DZ, Corollary of Theorem 2]. Moreover, in [DZ, Theorems 1-3], E. Hille's result is improved in terms of the asymptotic behaviour of the Cesàro means of $T$ and $T^{-1}$ as $n \rightarrow \infty$. Local results of Gelfand-Hille type can be found, for instance, in [AD], [Ze], [EZ], [D1], [D2]. We also recall that a characterization of nilpotency of $T$, in terms of the asymptotic behaviour of the positive iterates only of $I_{X}+T$ and $I_{X}-T$, 
is provided in [Al, Lemma 3]. Also [At] includes a characterization of nilpotency of $T-I_{X}$, in terms of the asymptotic behaviour of the positive iterates only of $T$ and of $T-I_{X}$ (see [At, Corollary 7]), as well as one of nilpotency of a quasinilpotent operator $A$, in terms of the growth of the resolvent function of $A$ (see [At, remark 3 on p. 50]). Characterizations of nilpotent operators by means of conditions on the growth of the resolvent function can also be found elsewhere in the literature ([Ge1, Theorem 1], see also [Na, III, $\S 11$, Theorem 9]; [Ne, 5.4.1]). Nilpotency of $T-I_{X}$, under the hypothesis $\sigma(T)=\{1\}$, is also characterized in [DZ, Theorem 4] by means of estimates of the norm of the resolvent function as $|\lambda| \rightarrow 1^{+}$and $|\lambda| \rightarrow 1^{-}$, and in [DZ, Theorem 5] by means of a generalization of the uniform Abel-boundedness condition on $T$ and $T^{-1}$. We also remark that necessary and sufficient conditions for nilpotency of a quasinilpotent operator $T$, in terms of finiteness of the ascent or descent of $T$, can be derived, as straightforward consequences, from the characterizations of the boundary points of the spectrum which are poles of the resolvent obtained by D. C. Lay in [L, 2.7 and 2.6, respectively] (for the consequence of [L, 2.6] see [TL, V, 10.6]; the consequence of [L, 2.7] will be explicitly recalled here, in the next section). Likewise, the refinements of D. C. Lay's results, provided by S. Grabiner in [Gr1, (5.4)] and [Gr2, (4.9)], and by S. Grabiner and J. Zemánek in [GZ, 2.3], yield corresponding refinements of the characterizations of nilpotency derived from [L, 2.7 and 2.6].

We are especially interested here in necessary and sufficient conditions for nilpotency of a bounded linear operator $T$ which involve closedness of the ranges of the (positive) iterates of $T$.

In [MZ], M. Mbekhta and J. Zemánek obtained an improvement of the uniform ergodic theorem, from which they derived a characterization of the identity operator among all the ones having spectrum equal to $\{1\}$, in terms of the asymptotic behaviour of the sequence $\left(T^{n}\right)_{n \in \mathbb{N}}$ as $n \rightarrow \infty$ and of closedness of the range of a suitable iterate of $I_{X}-T$ (see [MZ, Corollary 2]). Corollary 2 of [MZ] yields the following characterization of the zero operator among all the quasinilpotent ones, as a straightforward consequence (see also [MZ, Corollary 4]).

Let $X$ be a complex nonzero Banach space and $T \in L(X)$. Then $T=0_{L(X)}$ if and only if $\sigma(T)=\{0\}, n^{-1}\left\|\left(I_{X}-T\right)^{n}\right\|_{L(X)} \rightarrow 0$ as $n \rightarrow \infty$ and $\mathcal{R}\left(T^{m}\right)$ is closed for some $m \in \mathbb{Z}_{+}$.

The result below (which generalizes (1.1)) follows immediately from [LM, Corollary 7 and Lemma 3] (cf. also [GZ, 3.2 and (1.2b)]).

(1.2) Let $X$ and $T$ be as in (1.1), and $p \in \mathbb{Z}_{+}$. Then $T^{p}=0_{L(X)}$ if and only if $\sigma(T)=\{0\}, n^{-1}\left\|T^{p-1}\left(I_{X}-T\right)^{n}\right\|_{L(X)} \rightarrow 0$ as $n \rightarrow \infty$ and $\mathcal{R}\left(T^{m}\right)$ is closed for some $m \in \mathbb{N}$ satisfying $m \geq p$. 
In $[\mathrm{B} 2,3.4]$ we obtained a generalization of the uniform ergodic theorem to the sequence $\left(n^{-p} \sum_{k=0}^{n-1} T^{k}\right)_{n \in \mathbb{Z}_{+}}$(with $p \in \mathbb{Z}_{+}$), by giving several necessary and sufficient conditions in order that it converges in $L(X)$ and the subspace $\mathcal{R}\left(\left(I_{X}-T\right)^{p-1}\right)+\mathcal{N}\left(I_{X}-T\right)$ is closed (notice that, in the special case $p=1$, the latter condition is automatically satisfied, thus reducing the sum of the two conditions above to uniform ergodicity of $T$ ). A further condition, equivalent to the ones obtained in [B2], is provided in [B3, 3.4]. Also, in [B3, 3.5] a characterization of nilpotency of $T-I_{X}$ is derived from [B2, 3.4] and [B3, 3.4], yielding the following characterization of nilpotency of $T$ as a straightforward consequence.

Let $X$ be a complex nonzero Banach space, $T \in L(X)$ and $p \in \mathbb{Z}_{+}$. Then $T^{p}=0_{L(X)}$ if and only if $\sigma(T)=\{0\}, n^{-p}\left\|\left(I_{X}-T\right)^{n}\right\|_{L(X)} \rightarrow$ 0 as $n \rightarrow \infty$ and $\mathcal{R}\left(T^{k}\right)+\mathcal{N}\left(T^{j}\right)$ is closed for some $(k, j) \in \mathbb{Z}_{+} \times \mathbb{N}$ satisfying either $k=p$ and $j=0$ or $k+j>p$.

We recall that the condition $\lim _{n \rightarrow \infty} n^{-1}\left\|T^{p-1}\left(I_{X}-T\right)^{n}\right\|_{L(X)}=0$ implies $\lim _{n \rightarrow \infty} n^{-p}\left\|\left(I_{X}-T\right)^{n}\right\|_{L(X)}=0$ (see [B2, 2.4]), whereas the converse is not true for $p \geq 2$ (see [B2, 2.6]). Hence (1.3) improves (1.2) (cf. also [W, Theorem 4]). Also, it is known that convergence to zero in $L(X)$ (actually, even strong convergence to zero, see [W, Theorem 2]) of the sequence $\left(n^{-p}\left(I_{X}-T\right)^{n}\right)_{n \in \mathbb{Z}_{+}}$implies that the ascent of $T$ is less than or equal to $p$. Then from [GZ, 2.3] it follows that the norm convergence to zero requirement can be replaced by the weaker one that the ascent of $T$ be less than or equal to

$$
\begin{cases}1 & \text { in (1.1) for } m>1 \\ p & \text { in (1.2) for } m>p \text { and in (1.3) for } k+j>p .\end{cases}
$$

Notice that in the remaining cases (that is, for $m=1$ in (1.1), for $m=p$ in (1.2) and for $k=p, j=0$ in (1.3)) this is not true (see [B2, 3.5]); indeed, uniform convergence to zero of the sequence above cannot even be replaced by strong convergence to zero in these cases (see [GZ, 1.3]).

It is clear that eventual closedness of the ranges of the iterates of a bounded linear operator $T$ is a necessary condition in order that $T$ be nilpotent. The authors of [MZ] wonder whether, once quasinilpotency of $T$ is assumed, closedness of $\mathcal{R}\left(T^{n}\right)$ for $n$ large enough is also sufficient for nilpotency of $T$ (see the concluding remark 3 of [MZ]). This question can be answered in the negative. Indeed, in Theorem 3 of [Ap], C. Apostol proved that, if $\sigma$ is a (nonempty) countable compact set in the complex plane and $H$ is a complex infinite-dimensional Hilbert space, there exists $T \in L(H)$ such that $\sigma(T)=\sigma$ and $\mathfrak{p}(T)$ has closed range for all polynomials $\mathfrak{p}$. It turns out that the operator constructed in the proof of Theorem 3 of [Ap] is not nilpotent. Hence the proof of [Ap, Theorem 3], in the special case 
$\sigma=\{0\}$, yields an example of a nonnilpotent quasinilpotent bounded linear operator $T$ such that $\mathcal{R}\left(T^{n}\right)$ is closed for all $n \in \mathbb{N}$.

In this paper we provide several characterizations of nilpotency and construct another example showing that the question posed in [MZ] has a negative answer. We also use this example to give a negative answer to a further question, about sufficiency of two necessary conditions for nilpotency we derive here. In Section 2 we collect a few known results that are needed in the rest of the paper. In Section 3 we provide some necessary and sufficient conditions in order that a bounded linear operator has finite ascent (Theorem 3.6 and Corollary 3.7). These conditions are used in Section 4, in which several characterizations of nilpotency, for a bounded linear operator (Theorem 4.3) and for a quasinilpotent bounded linear operator (Theorem 4.7), are obtained. Also three necessary conditions for nilpotency ((4.4.1), (4.5.1) and (4.5.2)) are considered: (4.4.1) implies the remaining two, which are proved to be equivalent in Theorem 4.5. Most of our conditions for nilpotency involve closedness of the ranges (or of sums of ranges and kernels) of the iterates of the operator.

Section 5 is mainly devoted to examples. Indeed, in Example 5.4 we construct a nonnilpotent quasinilpotent bounded linear operator on an infinitedimensional Hilbert space, all the iterates of which have closed range. Actually, Example 5.4, which is the development of an example we had previously obtained (unpublished, although mentioned, together with another example by W. R. Wogen - about the same topic and unpublished as well - in the final comments of [Ze]), provides an infinite family of such operators, each of which corresponds to a square-summable sequence $\left(\varepsilon_{n}\right)_{n \in \mathbb{N}}$ of strictly positive numbers. We exactly compute the reduced minimum moduli of the iterates of these operators, in terms of the $\varepsilon_{n}$. We also give a formula to determine the norms of such iterates, besides providing upper estimates for them in terms of the $\varepsilon_{n}$. In Example 5.5 we use these computations to show that the sequence $\left(\varepsilon_{n}\right)_{n \in \mathbb{N}}$ can be chosen so that the operator of Example 5.4 satisfies (4.5.1) and (4.5.2), which therefore turn out not to be sufficient for nilpotency. In Remark 5.6 we show that Examples 5.4 and 5.5 leave open the question of sufficiency of condition (4.4.1), as it is not satisfied by the operator of Example 5.4 for any choice of $\left(\varepsilon_{n}\right)_{n \in \mathbb{N}}$. Finally, in Proposition 5.7, we remark that a result obtained in [Ap, Lemma 3] - that is, closedness of the range of $\mathfrak{p}(A)$ (where $A$ is a bounded linear operator on a complex Hilbert space $H$ ) for all polynomials $\mathfrak{p}$, under the hypothesis that $\mathcal{R}\left(\left(\lambda I_{H}-A\right)^{n}\right)$ is closed for all $\lambda \in \sigma(A)$ and $n \in \mathbb{N}$-actually holds in the more general setting of holomorphic functions of Banach space operators. This follows from a theorem-provided by M. Gonzalez and V. M. Onieva in [GO2] - which we are going to recall in Section 2, and clearly applies to the operator of Example 5.4, as well as to the operator constructed in the 
proof of [Ap, Theorem 3]: indeed, any holomorphic function of any of these two operators has closed range (Remark 5.8).

Acknowledgements. We wish to thank Warren Wogen for bringing Apostol's construction in Theorem 3 of [Ap] to our attention, and Jaroslav Zemánek for his interesting and stimulating questions and comments.

2. Preliminaries. Let $X$ be a Banach space. For every bounded linear operator $A$ on $X$, let $\alpha(A)$ denote the ascent of $A$, that is,

$$
\alpha(A)=\inf \left\{n \in \mathbb{N}: \mathcal{N}\left(A^{n}\right)=\mathcal{N}\left(A^{n+1}\right)\right\} .
$$

Notice that $\alpha(A) \in \mathbb{N} \cup\{\infty\}$ and the infimum above is attained if and only if $\alpha(A)<\infty$. Also, $\mathcal{N}\left(A^{n}\right) \subsetneq \mathcal{N}\left(A^{n+1}\right)$ for all $n \in \mathbb{N}$ satisfying $n<\alpha(A)$ and $\mathcal{N}\left(A^{n}\right)=\mathcal{N}\left(A^{n+1}\right)$ for $n \geq \alpha(A)$. Finally, the following equality is not difficult to verify.

$$
\text { If } A \text { is nilpotent, we have } \alpha(A)=\min \left\{p \in \mathbb{N}: A^{p}=0_{L(X)}\right\} \text {. }
$$

In what follows, we will need the following characterization of nilpotent operators, which is a consequence of $[\mathrm{L}, 2.7]$ and $[\mathrm{TL}, \mathrm{V}, 3.5,10.5$ and 10.6] (in these references, the Banach space is assumed to be complex and nonzero; anyway, the result holds in any Banach space, as the real nonzero case follows from the complex one by going to the complex extension of the operator, and the zero space case is trivial).

Theorem 2.2. Let $X$ be a Banach space and $T \in L(X)$ be quasinilpotent. Then $T$ is nilpotent if and only if $\alpha(T)<\infty$ and $\mathcal{R}\left(T^{n}\right)$ is closed for some $n \in \mathbb{Z}_{+}$satisfying $n \geq \alpha(T)+1$.

We will also use the following result by S. Grabiner and J. Zemánek.

Theorem 2.3 ([GZ, 2.1]). Let $X$ be a Banach space and $T \in L(X)$. If $\alpha(T)<\infty$ and $\mathcal{R}\left(T^{k}\right)+\mathcal{N}\left(T^{j}\right)$ is closed for some $(k, j) \in \mathbb{Z}_{+} \times \mathbb{N}$ satisfying $k+j \geq \alpha(T)+1$, then $\mathcal{R}\left(T^{n}\right)+\mathcal{N}\left(T^{m}\right)$ is closed for all $(n, m) \in \mathbb{N} \times \mathbb{N}$ satisfying $n+m \geq \alpha(T)$.

Notice that Theorem 2.3 yields a refinement of Theorem 2.2 in which closedness of $\mathcal{R}\left(T^{n}\right)$ for some $n \geq \alpha(T)+1$ can be replaced by closedness of $\mathcal{R}\left(T^{k}\right)+\mathcal{N}\left(T^{j}\right)$ for some $(k, j) \in \mathbb{Z}_{+} \times \mathbb{N}$ satisfying $k+j \geq \alpha(T)+1$ (a further refinement, in which also the condition $\alpha(T)<\infty$ is relaxed, follows from $[\mathrm{GZ}, 2.3])$.

For each nonempty subset $S$ of a normed space $Y$, let $\operatorname{dist}(\cdot, S)$ denote the distance function to $S$ on $Y$, that is,

$$
\operatorname{dist}(\cdot, S): Y \ni x \mapsto \operatorname{dist}(x, S)=\inf \left\{\|x-y\|_{Y}: y \in S\right\} \in \mathbb{R} .
$$


For every bounded linear operator $A$ on a Banach space $X$, let $\gamma(A)$ denote the reduced minimum modulus of $A$, that is,

$$
\gamma(A)=\sup \left\{m \in[0, \infty):\|A x\|_{X} \geq m \operatorname{dist}(x, \mathcal{N}(A)) \text { for all } x \in X\right\} .
$$

Notice that $\gamma(A) \in[0, \infty]$, and $\gamma(A)=\infty$ if and only if $A=0_{L(X)}$. We also recall that, if $A \neq 0_{L(X)}$, the supremum above is attained and $\gamma(A) \leq$ $\|A\|_{L(X)}$. The following well known result will be useful.

Theorem 2.4 ([K, IV , 5.2]; [TL, IV, 5.9]). Let $X$ be a Banach space and let $T \in L(X)$. Then $\mathcal{R}(T)$ is closed if and only if $\gamma(T)>0$.

The following result was obtained by C. Apostol in [Ap].

Theorem 2.5 ([Ap, Lemma 3]). Let $H$ be a complex Hilbert space and let $T \in L(H)$ be such that $\mathcal{R}\left(\left(\lambda I_{H}-T\right)^{n}\right)$ is closed for every $n \in \mathbb{N}$ and every $\lambda \in \sigma(T)$. Then $\mathcal{R}(\mathfrak{p}(T))$ is closed for every polynomial $\mathfrak{p}$.

The proof of Theorem 2.5 provided in [Ap] uses the fact (proved in [Ap, Corollary of Theorem 1]) that an operator $T \in L(H)$ such that $\mathcal{R}\left(\lambda I_{H}-T\right)$ is closed for every $\lambda \in \sigma(T)$ has an at most countable spectrum. We remark that this also holds in the general case of a Banach space operator (see [MO, Corollary 4.3 and Remark 4.4]). Hence the proof of [Ap, Lemma 3] can be repeated in the Banach space case. Anyway, the extension of Theorem 2.5 to the bounded linear operators on Banach spaces can also be obtained as a straightforward consequence of the fact that, if $\mathfrak{p}$ is the polynomial defined by $\mathfrak{p}(z)=\prod_{k=1}^{n}\left(\lambda_{k}-z\right)^{m_{k}}$ (where $n \in \mathbb{Z}_{+}, \lambda_{1}, \ldots, \lambda_{n} \in \mathbb{C}$, with $\lambda_{j} \neq \lambda_{h}$ for $j \neq h$, and $\left.m_{1}, \ldots, m_{n} \in \mathbb{Z}_{+}\right)$and $E$ is an endomorphism of a complex vector space $V$, we have $\mathcal{R}(\mathfrak{p}(E))=\bigcap_{k=1}^{n} \mathcal{R}\left(\left(\lambda_{k} I_{V}-E\right)^{m_{k}}\right)$ (see [Y, Theorem], or $[$ Go, Corollary] $)\left({ }^{1}\right)$.

Finally, we are going to recall a result which - as we will remark in Proposition 5.7-yields the extension of Theorem 2.5 to holomorphic functions of (Banach space) operators.

If $X$ is a complex Banach space and $T \in L(X)$, let $\mathcal{U}(T)$ denote the set of all complex-valued holomorphic functions whose domain is a nonempty open subset of $\mathbb{C}$ containing $\sigma(T)$. We recall (see for instance [TL, Definition on p. 310 and subsequent comments]) that, for each $f \in \mathcal{U}(T)$, the operator

$\left({ }^{1}\right)$ We recall that this result also holds for non-everywhere defined operators: indeed, if $\mathfrak{p}$ and $\mathfrak{q}$ are polynomials with no common roots in $\mathbb{C}$ and $A$ is a (possibly non-everywhere defined) linear operator on a complex vector space, then $\mathcal{R}(\mathfrak{p q}(A))=\mathcal{R}(\mathfrak{p}(A)) \cap \mathcal{R}(\mathfrak{q}(A))$. This-together with the equality $\mathcal{N}(\mathfrak{p}(A))=\mathfrak{q}(A)(\mathcal{N}(\mathfrak{p}(A)))$ - can be found, for instance, in $[B 1,2.2]$. We take this opportunity to point out that a different proof of the results of $[\mathrm{B} 1,2.2]$ had been previously provided in [GO1] (see [GO1, (1.1) and (1.3)]). 
$f(T) \in L(X)$ is defined by

$$
f(T)=\frac{1}{2 \pi i} \int_{+\partial D} f(z) R(z, T) d z,
$$

where $D$ is any nonempty open bounded subset of $\mathbb{C}$ containing $\sigma(T)$ and with closure contained in the domain of $f$, which has finitely many components with pairwise disjoint closures, and whose boundary consists of a finite number of simple closed rectifiable curves, no two of which intersect and each of which is positively oriented with respect to $D ;+\partial D$ denotes the positively oriented boundary of $D$. This extends the canonical definition of $\mathfrak{p}(T)$, where $\mathfrak{p}$ is a polynomial with coefficients in $\mathbb{C}$ (see [TL, V, 8.1]).

Theorem 2.6 (see [GO2, Theorem]). Let $X$ be a complex Banach space, $T \in L(X)$ and $f \in \mathcal{U}(T)$. For each isolated zero $\mu$ of $f$, let $m_{\mu}$ denote the order of $\mu$. Then $\mathcal{R}(f(T))$ is closed if and only if $\mathcal{R}\left(\left(\lambda I_{X}-T\right)^{m_{\lambda}}\right)$ is closed for every isolated zero $\lambda$ of $f$ satisfying $\lambda \in \sigma(T)$.

3. Characterizations of operators with finite ascent. We remark that, if $V$ is a linear space, $I$ is a nonempty set and $\left(p_{i}\right)_{i \in I}$ is a family of seminorms on $V$, the set $W$ of all $x \in V$ satisfying $\sum_{i \in I} p_{i}(x)<\infty$ is a linear subspace of $V$. Notice also that the function $q: W \ni x \mapsto \sum_{i \in I} p_{i}(x) \in \mathbb{R}$ is a seminorm on $W$. Moreover, $q$ is a norm if the set $\left\{p_{i}: i \in I\right\}$ contains a norm.

Definition 3.1. Let $X$ be a normed space, let $I$ be a nonempty set and let $\mathcal{P}=\left(p_{i}\right)_{i \in I}$ be a family of seminorms on $X$ such that \|\|$_{X} \in\left\{p_{i}: i \in I\right\}$. We will denote by $X_{\mathcal{P}}$ the space of all $x \in X$ satisfying $\sum_{i \in I} p_{i}(x)<\infty$, endowed with the norm \|\|$_{X_{\mathcal{P}}}$ defined by

$$
\|x\|_{X_{\mathcal{P}}}=\sum_{i \in I} p_{i}(x) \quad \text { for every } x \in X_{\mathcal{P}}
$$

We remark that $X_{\mathcal{P}}$ is continuously embedded in $X$, since \|\|$_{X} \in\left\{p_{i}\right.$ : $i \in I\}$ and consequently $\|x\|_{X} \leq\|x\|_{X_{\mathcal{P}}}$ for each $x \in X_{\mathcal{P}}$.

Lemma 3.2. Let $X$ be a Banach space, let $I$ be a nonempty set and let $\mathcal{P}=\left(p_{i}\right)_{i \in I}$ be a family of continuous seminorms on $X$ such that \|\|$_{X} \in$ $\left\{p_{i}: i \in I\right\}$. Then $X_{\mathcal{P}}$ is a Banach space.

Proof. Let $\left(x_{n}\right)_{n \in \mathbb{N}}$ be a Cauchy sequence in $X_{\mathcal{P}}$. Since $\|x\|_{X} \leq\|x\|_{X_{\mathcal{P}}}$ for each $x \in X_{\mathcal{P}}$, it follows that $\left(x_{n}\right)_{n \in \mathbb{N}}$ is also a Cauchy sequence in $X$. Hence there exists $x \in X$ such that $x_{n} \stackrel{X}{\longrightarrow} x$ as $n \rightarrow \infty$. We prove that $x \in X_{\mathcal{P}}$ and $x_{n} \stackrel{X_{\mathcal{P}}}{\longrightarrow} x$ as $n \rightarrow \infty$. 
Fix $\varepsilon>0$. Since $\left(x_{n}\right)_{n \in \mathbb{N}}$ is a Cauchy sequence in $X_{\mathcal{P}}$, there exists $\nu_{\varepsilon} \in \mathbb{N}$ such that

$$
\sum_{i \in I} p_{i}\left(x_{n}-x_{m}\right)<\varepsilon \quad \text { for all } n, m \in \mathbb{N} \text { satisfying } n, m \geq \nu_{\varepsilon} .
$$

Now let $n \in \mathbb{N}$ be such that $n \geq \nu_{\varepsilon}$. For each nonempty finite subset $F$ of $I$, we have

$$
\sum_{i \in F} p_{i}\left(x_{n}-x_{m}\right) \leq \sum_{i \in I} p_{i}\left(x_{n}-x_{m}\right)<\varepsilon \quad \text { for all } m \geq \nu_{\varepsilon},
$$

which, since the elements of $\left\{p_{i}: i \in I\right\}$ are continuous on $X$, yields

$$
\sum_{i \in F} p_{i}\left(x_{n}-x\right) \leq \varepsilon
$$

If $\mathcal{F}_{I}$ denotes the set of all nonempty finite subsets of $I$, we conclude that

$$
\sum_{i \in I} p_{i}\left(x_{n}-x\right)=\sup \left\{\sum_{i \in F} p_{i}\left(x_{n}-x\right): F \in \mathcal{F}_{I}\right\} \leq \varepsilon \quad \text { for all } n \geq \nu_{\varepsilon} .
$$

Hence $x_{n}-x \in X_{\mathcal{P}}$ for some $n \in \mathbb{N}$, and consequently $x \in X_{\mathcal{P}}$. Furthermore,

$$
\left\|x_{n}-x\right\|_{X_{\mathcal{P}}}=\sum_{i \in I} p_{i}\left(x_{n}-x\right) \rightarrow 0 \quad \text { as } n \rightarrow \infty,
$$

which gives the desired result.

If not all of the seminorms of the family $\mathcal{P}$ are continuous on $X$, the normed space $X_{\mathcal{P}}$ may not be complete, as the following example shows.

Example 3.3. Let $X$ be an infinite-dimensional Banach space. Then there exists a complete norm $\mathfrak{n}$ on $X$ which is not equivalent to \|\|$_{X}$. For instance, $\mathfrak{n}$ can be defined by

$$
\mathfrak{n}(x)=\|T x\|_{X} \quad \text { for every } x \in X,
$$

where $T$ is any one-to-one discontinuous linear operator from $X$ into $X$, having closed range in $X$ (such an operator exists, as $X$ has infinite dimension): indeed, $\mathfrak{n}$ is a norm as $T$ is linear and one-to-one; furthermore, the normed space $(X, \mathfrak{n})$ is complete as the range of $T$ is closed in $\left(X,\|\|_{X}\right)$.

Let $\mathcal{S}$ be the couple of seminorms on $X$ defined by $\mathcal{S}=\left(\mathfrak{n},\|\|_{X}\right)$. Then the linear subspace $X_{\mathcal{S}}$ coincides with the whole of $X$ and

$$
\|x\|_{X_{\mathcal{S}}}=\mathfrak{n}(x)+\|x\|_{X} \quad \text { for every } x \in X .
$$

Notice that $X_{\mathcal{S}}$ is continuously embedded in $(X, \mathfrak{n})$ as well as in $\left(X,\|\|_{X}\right)$. Since the identity map from $\left(X,\|\|_{X}\right)$ onto $(X, \mathfrak{n})$ is discontinuous and both spaces are Banach, from the closed graph theorem it follows that there exist a sequence $\left(x_{n}\right)_{n \in \mathbb{N}}$ in $X$ and $x, y \in X$ such that $\left(x_{n}\right)_{n \in \mathbb{N}}$ converges to $x$ in $\left(X,\|\|_{X}\right)$ and to $y$ in $(X, \mathfrak{n})$, and $x \neq y$. Then $\left(x_{n}\right)_{n \in \mathbb{N}}$ is a Cauchy sequence in both $\left(X,\|\|_{X}\right)$ and $(X, \mathfrak{n})$, and consequently it is a Cauchy sequence 
in $X_{\mathcal{S}}$. Nevertheless, since $X_{\mathcal{S}}$ is continuously embedded in both $\left(X,\|\|_{X}\right)$ and $(X, \mathfrak{n})$ and $x \neq y$, it follows that $\left(x_{n}\right)_{n \in \mathbb{N}}$ does not converge in $X_{\mathcal{S}}$. Hence $X_{\mathcal{S}}$ is not complete.

Let $S$ be a nonempty subset of a normed space $Y$. We remark that $\operatorname{dist}(\cdot, S)$ is continuous on $Y$, as

$$
|\operatorname{dist}(x, S)-\operatorname{dist}(y, S)| \leq\|x-y\|_{Y} \quad \text { for all } x, y \in Y \text {. }
$$

Furthermore, if $S$ is a linear subspace of $Y$, then $\operatorname{dist}(\cdot, S)$ is a seminorm on $Y$.

Definition 3.4. Let $X$ be a Banach space, let $T \in L(X)$ and let $\Sigma$ be a subset of $\mathbb{N}$ containing 0 . We set

$$
\mathcal{D}(\Sigma, T)=\left(\operatorname{dist}\left(\cdot, \mathcal{N}\left(T^{n}\right)\right)\right)_{n \in \Sigma} .
$$

Under the hypotheses of Definition 3.4, $\mathcal{D}(\Sigma, T)$ is a family of continuous seminorms on $X$; furthermore, \|\|$_{X}=\operatorname{dist}\left(\cdot, \mathcal{N}\left(T^{0}\right)\right) \in\left\{\operatorname{dist}\left(\cdot, \mathcal{N}\left(T^{n}\right)\right)\right.$ : $n \in \Sigma$ \}. Thus $X_{\mathcal{D}(\Sigma, T)}$ is a Banach space by Lemma 3.2.

For every element $y$ of a normed space $Y$ and for every $\varepsilon>0$, let $B_{Y}(y, \varepsilon)$ stand for the open ball in $Y$ centered at $y$ with radius $\varepsilon$.

Lemma 3.5. Let $X$ be a Banach space, let $T \in L(X)$ and let $\Sigma$ be an infinite subset of $\mathbb{N}$ containing 0 . Then $X_{\mathcal{D}(\Sigma, T)} \subset \overline{\bigcup_{n \in \mathbb{N}} \mathcal{N}\left(T^{n}\right)}$.

Proof. For each $x \in X \backslash \overline{\bigcup_{n \in \mathbb{N}} \mathcal{N}\left(T^{n}\right)}$, there exists $\delta>0$ such that $B_{X}(x, \delta) \cap \bigcup_{n \in \mathbb{N}} \mathcal{N}\left(T^{n}\right)=\emptyset$. Hence $\operatorname{dist}\left(x, \mathcal{N}\left(T^{n}\right)\right) \geq \delta$ for all $n \in \mathbb{N}$, which, since $\Sigma$ is infinite, gives $\sum_{n \in \Sigma} \operatorname{dist}\left(x, \mathcal{N}\left(T^{n}\right)\right)=\infty$.

Theorem 3.6. Let $X$ be a Banach space and let $T \in L(X)$. The following conditions are equivalent:

(3.6.2) there exists $M \in[0, \infty)$ such that $\sum_{n=0}^{\infty} \operatorname{dist}\left(x, \mathcal{N}\left(T^{n}\right)\right) \leq M\|x\|_{X}$ for every $x \in \overline{\bigcup_{n \in \mathbb{N}} \mathcal{N}\left(T^{n}\right)}$;

$$
\begin{aligned}
& \sum_{n=0}^{\infty} \operatorname{dist}\left(x, \mathcal{N}\left(T^{n}\right)\right)<\infty \text { for every } x \in \overline{\bigcup_{n \in \mathbb{N}} \mathcal{N}\left(T^{n}\right)} ; \\
& \text { there exists a strictly increasing sequence }\left(n_{k}\right)_{k \in \mathbb{N}} \text { of nonnega- } \\
& \text { tive integers such that } \sum_{k=0}^{\infty} \operatorname{dist}\left(x, \mathcal{N}\left(T^{n_{k}}\right)\right)<\infty \text { for every } x \in \\
& \bigcup_{n \in \mathbb{N}} \mathcal{N}\left(T^{n}\right) .
\end{aligned}
$$

Furthermore, if (3.6.1)-(3.6.4) are satisfied, we have

$$
\begin{aligned}
\alpha(T)=\min \left\{M \in[0, \infty): \sum_{n=0}^{\infty} \operatorname{dist}\left(x, \mathcal{N}\left(T^{n}\right)\right) \leq M\|x\|_{X}\right. \\
\left.\quad \text { for every } x \in \overline{\bigcup_{n \in \mathbb{N}} \mathcal{N}\left(T^{n}\right)}\right\} .
\end{aligned}
$$


Proof. We begin by supposing that $\alpha(T)<\infty$. We prove that then

$$
\sum_{n=0}^{\infty} \operatorname{dist}\left(x, \mathcal{N}\left(T^{n}\right)\right) \leq \alpha(T)\|x\|_{X} \quad \text { for every } x \in \overline{\bigcup_{n \in \mathbb{N}} \mathcal{N}\left(T^{n}\right)} .
$$

This is clearly true if $\alpha(T)=0$. Now assume $\alpha(T) \in \mathbb{Z}_{+}$. Then, for each $x \in \overline{\bigcup_{n \in \mathbb{N}} \mathcal{N}\left(T^{n}\right)}=\mathcal{N}\left(T^{\alpha(T)}\right)$, we have

$$
\sum_{n=0}^{\infty} \operatorname{dist}\left(x, \mathcal{N}\left(T^{n}\right)\right)=\sum_{n=0}^{\alpha(T)-1} \operatorname{dist}\left(x, \mathcal{N}\left(T^{n}\right)\right) \leq \sum_{n=0}^{\alpha(T)-1}\|x\|_{X}=\alpha(T)\|x\|_{X},
$$

which is the desired result.

We have thus proved that (3.6.1) $\Rightarrow(3.6 .2)$. Clearly, (3.6.2) $\Rightarrow(3.6 .3)$ and $(3.6 .3) \Rightarrow(3.6 .4)$. Now we prove that $(3.6 .4) \Rightarrow(3.6 .1)$.

Suppose condition (3.6.4) holds and define $\Sigma=\{0\} \cup\left\{n_{k}: k \in \mathbb{N}\right\}$. Then $\mathcal{D}(\Sigma, T)$ is a family of continuous seminorms on $X$ and \|\|$_{X} \in$ $\left\{\operatorname{dist}\left(\cdot, \mathcal{N}\left(T^{n}\right)\right): n \in \Sigma\right\}$. Moreover, $X_{\mathcal{D}(\Sigma, T)} \subset \overline{\bigcup_{n \in \mathbb{N}} \mathcal{N}\left(T^{n}\right)}$ by Lemma 3.5. Condition (3.6.4) gives the opposite inclusion, so $X_{\mathcal{D}(\Sigma, T)}=\overline{\bigcup_{n \in \mathbb{N}} \mathcal{N}\left(T^{n}\right)}$. Since $X_{\mathcal{D}(\Sigma, T)}$ is continuously embedded in $X$ and \|\|$_{X_{\mathcal{D}(\Sigma, T)}}$ is complete by Lemma 3.2, from the open mapping theorem we conclude that the norms \|\|$_{X_{\mathcal{D}(\Sigma, T)}}$ and \|\|$_{X}$ are equivalent on $X_{\mathcal{D}(\Sigma, T)}$. Hence there exists $M \in$ $(0, \infty)$ such that $\|x\|_{X_{\mathcal{D}(\Sigma, T)}} \leq M\|x\|_{X}$ for every $x \in X_{\mathcal{D}(\Sigma, T)}$, which gives

$$
\sum_{k=0}^{\infty} \operatorname{dist}\left(x, \mathcal{N}\left(T^{n_{k}}\right)\right) \leq M\|x\|_{X} \quad \text { for every } x \in \overline{\bigcup_{n \in \mathbb{N}} \mathcal{N}\left(T^{n}\right)} .
$$

We now prove that $\alpha(T)<\infty$. If the ascent of $T$ were infinite, for every $k \in \mathbb{N}$ we would have $\mathcal{N}\left(T^{n_{k}}\right) \subsetneq \mathcal{N}\left(T^{n_{k+1}}\right)$. Hence, by Riesz's lemma, there would exist $x_{k} \in \mathcal{N}\left(T^{n_{k+1}}\right)$ such that $\left\|x_{k}\right\|_{X}=1$ and $\operatorname{dist}\left(x_{k}, \mathcal{N}\left(T^{n_{k}}\right)\right) \geq 1 / 2$. Then from (3.6.6) we would obtain

$$
\begin{aligned}
M & =M\left\|x_{k}\right\|_{X} \geq \sum_{j=0}^{\infty} \operatorname{dist}\left(x_{k}, \mathcal{N}\left(T^{n_{j}}\right)\right)=\sum_{j=0}^{k} \operatorname{dist}\left(x_{k}, \mathcal{N}\left(T^{n_{j}}\right)\right) \\
& \geq(k+1) / 2 \quad \text { for all } k \in \mathbb{N},
\end{aligned}
$$

which is impossible. Hence $\alpha(T)<\infty$.

We have thus proved that conditions (3.6.1)-(3.6.4) are equivalent. Now suppose they are satisfied and set

$$
\begin{aligned}
\mathfrak{M}=\left\{M \in[0, \infty): \sum_{n=0}^{\infty} \operatorname{dist}\left(x, \mathcal{N}\left(T^{n}\right)\right) \leq\right. & M\|x\|_{X} \\
& \left.\quad \text { for every } x \in \overline{\bigcup_{n \in \mathbb{N}} \mathcal{N}\left(T^{n}\right)}\right\} .
\end{aligned}
$$


We will prove that $\alpha(T)=\min (\mathfrak{M})$. From (3.6.5) it follows that $\alpha(T) \in \mathfrak{M}$, which gives the desired result if $\alpha(T)=0$. Suppose $\alpha(T) \in \mathbb{Z}_{+}$and let $M \in \mathfrak{M}$. By Riesz's lemma, for each $\varepsilon \in(0,1)$ there exists $x_{\varepsilon} \in \mathcal{N}\left(T^{\alpha(T)}\right)$ such that $\left\|x_{\varepsilon}\right\|_{X}=1$ and $\operatorname{dist}\left(x_{\varepsilon}, \mathcal{N}\left(T^{\alpha(T)-1}\right)\right) \geq 1-\varepsilon$. Hence

$$
\begin{aligned}
M & =M\left\|x_{\varepsilon}\right\|_{X} \geq \sum_{n=0}^{\infty} \operatorname{dist}\left(x_{\varepsilon}, \mathcal{N}\left(T^{n}\right)\right)=\sum_{n=0}^{\alpha(T)-1} \operatorname{dist}\left(x_{\varepsilon}, \mathcal{N}\left(T^{n}\right)\right) \\
& \geq(1-\varepsilon) \alpha(T) .
\end{aligned}
$$

Letting $\varepsilon \rightarrow 1^{-}$, we obtain $M \geq \alpha(T)$. Hence $\alpha(T)=\min (\mathfrak{M})$, which finishes the proof of the theorem.

Henceforth, we stipulate that $\lambda / \infty=0$ for all $\lambda \in[0, \infty)$. This, in virtue of Theorem 2.4, allows us to consider $\|A x\|_{X} / \gamma(A)$ for any $A \in L(X)$ having closed range in $X$ (where $X$ is any Banach space) and for every $x \in X$.

Corollary 3.7. Let $X$ be a Banach space, $T \in L(X)$ and $\left(n_{k}\right)_{k \in \mathbb{N}}$ be a strictly increasing sequence of nonnegative integers such that $\mathcal{R}\left(T^{n_{k}}\right)$ is closed for all $k \in \mathbb{N}$. Then:

$$
\begin{aligned}
& \sum_{k=0}^{\infty} \operatorname{dist}\left(x, \mathcal{N}\left(T^{n_{k}}\right)\right) \leq \sum_{k=0}^{\infty}\left\|T^{n_{k}} x\right\|_{X} / \gamma\left(T^{n_{k}}\right) \text { for every } x \in X ; \\
& \alpha(T)<\infty \text { if and only if } \sum_{k=0}^{\infty}\left\|T^{n_{k}} x\right\|_{X} / \gamma\left(T^{n_{k}}\right)<\infty \text { for every } \\
& x \in \overline{\bigcup_{n \in \mathbb{N}} \mathcal{N}\left(T^{n}\right)} .
\end{aligned}
$$

Proof. (3.7.1) follows from the properties of the reduced minimum modulus, and the "if" part of (3.7.2) follows from (3.7.1) and Theorem 3.6.

Now we prove the "only if" part of (3.7.2). If $\alpha(T)<\infty$, we have $\overline{\bigcup_{n \in \mathbb{N}} \mathcal{N}\left(T^{n}\right)}=\bigcup_{n \in \mathbb{N}} \mathcal{N}\left(T^{n}\right)$. Then, for each $x \in \overline{\bigcup_{n \in \mathbb{N}} \mathcal{N}\left(T^{n}\right)}$, the series $\sum_{k=0}^{\infty}\left\|T^{n_{k}} x\right\|_{X} / \gamma\left(T^{n_{k}}\right)$ reduces to a finite sum and hence converges. The corollary is proved.

4. Characterizations of nilpotent operators. In this section, we are going to use Theorem 3.6 in order to derive characterizations of nilpotency for a bounded linear operator on a Banach space.

Definition 4.1. Let $X$ be a Banach space and let $T \in L(X)$. We set $\kappa(T)=\inf \left\{n \in \mathbb{Z}_{+}: \mathcal{R}\left(T^{k}\right)\right.$ is closed for all $k \in \mathbb{N}$ satisfying $\left.k \geq n\right\}$.

We remark that $\kappa(T) \in \mathbb{Z}_{+} \cup\{\infty\}$ and the infimum above is attained if and only if $\kappa(T)<\infty$. Notice also that $\kappa(T)<\infty$ if and only if $\mathcal{R}\left(T^{n}\right)$ is eventually closed. In particular, $\kappa(T)=1$ if and only if $\mathcal{R}\left(T^{n}\right)$ is closed for all $n \in \mathbb{N}$.

We will need the following elementary result. 
Lemma 4.2. Let $X$ be a Banach space and let $T \in L(X)$. Then $T$ is nilpotent if and only if $\alpha(T)<\infty$ and $\bigcup_{n \in \mathbb{N}} \mathcal{N}\left(T^{n}\right)$ is dense in $X$.

Proof. If $\alpha(T)<\infty$ and $\bigcup_{n \in \mathbb{N}} \mathcal{N}\left(T^{n}\right)$ is dense in $X$, we have

$$
X=\overline{\bigcup_{n \in \mathbb{N}} \mathcal{N}\left(T^{n}\right)}=\mathcal{N}\left(T^{\alpha(T)}\right),
$$

which gives $T^{\alpha(T)}=0_{L(X)}$. Hence $T$ is nilpotent. The converse follows from (2.1).

Theorem 4.3. Let $X$ be a Banach space and let $T \in L(X)$. The following conditions are equivalent:

(4.3.1) $T$ is nilpotent;

(4.3.2) there exists $M \in[0, \infty)$ such that $\sum_{n=0}^{\infty} \operatorname{dist}\left(x, \mathcal{N}\left(T^{n}\right)\right) \leq M\|x\|_{X}$ for every $x \in X$;

(4.3.3) $\quad \sum_{n=0}^{\infty} \operatorname{dist}\left(x, \mathcal{N}\left(T^{n}\right)\right)<\infty$ for every $x \in X$;

(4.3.4) there exists a strictly increasing sequence $\left(n_{k}\right)_{k \in \mathbb{N}}$ of nonnegative integers such that $\sum_{k=0}^{\infty} \operatorname{dist}\left(x, \mathcal{N}\left(T^{n_{k}}\right)\right)<\infty$ for every $x \in X$;

(4.3.5) $\quad \kappa(T)<\infty$ and there exists $N \in[0, \infty)$ such that

$$
\sum_{n=\kappa(T)}^{\infty}\left\|T^{n} x\right\|_{X} / \gamma\left(T^{n}\right) \leq N\|x\|_{X} \quad \text { for every } x \in X
$$

$$
\kappa(T)<\infty \text { and } \sum_{n=\kappa(T)}^{\infty}\left\|T^{n} x\right\|_{X} / \gamma\left(T^{n}\right)<\infty \text { for every } x \in X ;
$$

there exists a strictly increasing sequence $\left(n_{k}\right)_{k \in \mathbb{N}}$ of nonnegative integers such that $\mathcal{R}\left(T^{n_{k}}\right)$ is closed for all $k \in \mathbb{N}$ and

$$
\sum_{k=0}^{\infty}\left\|T^{n_{k}} x\right\|_{X} / \gamma\left(T^{n_{k}}\right)<\infty \quad \text { for every } x \in X .
$$

Furthermore, if (4.3.1)-(4.3.7) are satisfied, we have

$$
=\min \left\{M \in[0, \infty): \sum_{n=0}^{\infty} \operatorname{dist}\left(x, \mathcal{N}\left(T^{n}\right)\right) \leq M\|x\|_{X} \text { for every } x \in X\right\} .
$$

Proof. From Lemma 4.2, Theorem 3.6, Lemma 3.5 and (2.1) we derive that conditions (4.3.1)-(4.3.4) are equivalent and (4.3.8) holds if they are satisfied.

Now suppose that (4.3.1)-(4.3.4) are satisfied. Then there exists $p \in \mathbb{Z}_{+}$ such that $T^{p}=0_{L(X)}$. Hence $\kappa(T) \leq p$. Furthermore, for each $x \in X$, we 
have

$$
\sum_{n=\kappa(T)}^{\infty} \frac{\left\|T^{n} x\right\|_{X}}{\gamma\left(T^{n}\right)}=\sum_{n=\kappa(T)}^{p} \frac{\left\|T^{n} x\right\|_{X}}{\gamma\left(T^{n}\right)} \leq\left(\sum_{n=\kappa(T)}^{p} \frac{\left\|T^{n}\right\|_{L(X)}}{\gamma\left(T^{n}\right)}\right)\|x\|_{X},
$$

which gives (4.3.5).

We have thus proved that (4.3.1)-(4.3.4) imply (4.3.5). Clearly, we have $(4.3 .5) \Rightarrow(4.3 .6)$ and $(4.3 .6) \Rightarrow(4.3 .7)$. Furthermore, by (3.7.1), condition (4.3.7) implies (4.3.4), which completes the proof.

The next result follows from Theorem 4.3 (more precisely, from equivalence between (4.3.1) and (4.3.6)). It can also be checked directly by observing that $\mathcal{R}\left(T^{n}\right)$ is eventually closed and the series $\sum_{n=\kappa(T)}^{\infty} T^{n} x / \gamma\left(T^{n}\right)$ reduces to a finite sum for every $x \in X$ if $T$ is nilpotent.

Proposition 4.4. Let $X$ be a Banach space and $T \in L(X)$ be nilpotent. Then

(4.4.1) $T$ is quasinilpotent, $\kappa(T)<\infty$ and the series $\sum_{n=\kappa(T)}^{\infty} T^{n} x / \gamma\left(T^{n}\right)$ converges in $X$ for every $x \in X$.

TheOrem 4.5. Let $X$ be a Banach space and $T \in L(X)$. Then the following two conditions are equivalent.

(4.5.1) $T$ is quasinilpotent, $\kappa(T)<\infty$ and $\lim _{n \rightarrow \infty} T^{n} x / \gamma\left(T^{n}\right)=0_{X}$ for every $x \in X$;

(4.5.2) $T$ is quasinilpotent, $\bigcup_{n \in \mathbb{N}} \mathcal{N}\left(T^{n}\right)$ is dense in $X, \kappa(T)<\infty$ and the sequence $\left(\left\|T^{n}\right\|_{L(X)} / \gamma\left(T^{n}\right)\right)_{n \geq \kappa(T)}$ is bounded.

Furthermore, condition (4.4.1) implies (4.5.1) and (4.5.2).

Proof. Clearly, (4.4.1) implies (4.5.1). If $T$ satisfies (4.5.1), we have

$$
\lim _{n \rightarrow \infty}\left\|T^{n} x\right\|_{X} / \gamma\left(T^{n}\right)=0 \quad \text { for every } x \in X .
$$

For each $x \in X$, since

$$
\operatorname{dist}\left(x, \mathcal{N}\left(T^{n}\right)\right) \leq\left\|T^{n} x\right\|_{X} / \gamma\left(T^{n}\right) \quad \text { for every } n \geq \kappa(T),
$$

from (4.5.3) we conclude that $\lim _{n \rightarrow \infty} \operatorname{dist}\left(x, \mathcal{N}\left(T^{n}\right)\right)=0$, which gives $x \in$ $\overline{\bigcup_{n \in \mathbb{N}} \mathcal{N}\left(T^{n}\right)}$. Hence $\overline{\bigcup_{n \in \mathbb{N}} \mathcal{N}\left(T^{n}\right)}=X$.

From (4.5.3) it also follows that the sequence $\left(\left\|T^{n} x\right\|_{X} / \gamma\left(T^{n}\right)\right)_{n \geq \kappa(T)}$ is bounded for every $x \in X$. By the uniform boundedness principle, this yields boundedness of $\left(\left\|T^{n}\right\|_{L(X)} / \gamma\left(T^{n}\right)\right)_{n \geq \kappa(T)}$. We have thus proved that (4.5.1) implies (4.5.2).

Conversely, suppose (4.5.2) is satisfied and let $M \in[0, \infty)$ be such that

$$
\left\|T^{n}\right\|_{L(X)} / \gamma\left(T^{n}\right) \leq M \quad \text { for all } n \geq \kappa(T) .
$$


Fix $x \in X$. Since $\bigcup_{n \in \mathbb{N}} \mathcal{N}\left(T^{n}\right)$ is dense in $X$, we conclude that

$$
\lim _{n \rightarrow \infty} \operatorname{dist}\left(x, \mathcal{N}\left(T^{n}\right)\right)=\operatorname{dist}\left(x, \bigcup_{n \in \mathbb{N}} \mathcal{N}\left(T^{n}\right)\right)=0 .
$$

Since, by (4.5.4), for each $n \geq \kappa(T)$ we have

$$
\frac{\left\|T^{n} x\right\|_{X}}{\gamma\left(T^{n}\right)} \leq \frac{\left\|T^{n}\right\|_{L(X)}}{\gamma\left(T^{n}\right)} \operatorname{dist}\left(x, \mathcal{N}\left(T^{n}\right)\right) \leq M \operatorname{dist}\left(x, \mathcal{N}\left(T^{n}\right)\right),
$$

from (4.5.5) it follows that $\lim _{n \rightarrow \infty} T^{n} x / \gamma\left(T^{n}\right)=0_{X}$. Hence $T$ satisfies (4.5.1). We have thus proved that (4.5.1) and (4.5.2) are equivalent. The proof is now complete.

We remark that the requirement that $T$ be quasinilpotent cannot be removed from conditions (4.5.1) and (4.5.2) without weakening them. Indeed, for instance, the backward shift operator $S$ on $\ell_{2}$ satisfies $\kappa(S)=1$ and $\lim _{n \rightarrow \infty} S^{n} x / \gamma\left(S^{n}\right)=\lim _{n \rightarrow \infty} S^{n} x=0_{\ell_{2}}$ for every $x \in \ell_{2}$. Nevertheless, $S$ is not quasinilpotent.

The next result follows from Proposition 4.4 and Theorem 4.5. It can as well be checked directly, by observing that $\mathcal{R}\left(T^{n}\right)$ is eventually closed and the sequence $\left(T^{n} x / \gamma\left(T^{n}\right)\right)_{n \geq \kappa(T)}$ is eventually zero for every $x \in X$ if $T$ is nilpotent.

Proposition 4.6. Let $X$ be a Banach space and $T \in L(X)$ be nilpotent. Then $T$ satisfies the equivalent conditions (4.5.1) and (4.5.2).

In the next section, we will show that (4.5.1) and (4.5.2) are not sufficient for nilpotency of $T$.

Henceforth, $[x]$ will denote the integer part of $x$ for every $x \in \mathbb{R}$.

Theorem 4.7. Let $X$ be a Banach space and $T \in L(X)$ be quasinilpotent. The following conditions are equivalent:

(4.7.1) $T$ is nilpotent;

(4.7.2) there exists $M \in[0, \infty)$ such that $\sum_{n=0}^{\infty} \operatorname{dist}\left(x, \mathcal{N}\left(T^{n}\right)\right) \leq M\|x\|_{X}$ for every $x \in \overline{\bigcup_{n \in \mathbb{N}} \mathcal{N}\left(T^{n}\right)}$ and $\mathcal{R}\left(T^{k}\right)+\mathcal{N}\left(T^{j}\right)$ is closed for some $(k, j) \in \mathbb{Z}_{+} \times \mathbb{N}$ satisfying $k+j \geq[M]+1$

(4.7.3) there exists $M \in[0, \infty)$ such that $\sum_{n=0}^{\infty} \operatorname{dist}\left(x, \mathcal{N}\left(T^{n}\right)\right) \leq M\|x\|_{X}$ for every $x \in \overline{\bigcup_{n \in \mathbb{N}} \mathcal{N}\left(T^{n}\right)}$ and $\mathcal{R}\left(T^{k}\right)+\mathcal{N}\left(T^{j}\right)$ is closed for all $(k, j) \in \mathbb{N} \times \mathbb{N}$ satisfying $k+j \geq[M]$

(4.7.4) $\quad \sum_{n=0}^{\infty} \operatorname{dist}\left(x, \mathcal{N}\left(T^{n}\right)\right)<\infty$ for all $x \in \overline{\bigcup_{n \in \mathbb{N}} \mathcal{N}\left(T^{n}\right)}$ and $\mathcal{R}\left(T^{k}\right)+$ $\mathcal{N}\left(T^{j}\right)$ is closed for infinitely many $(k, j) \in \mathbb{Z}_{+} \times \mathbb{N}$;

(4.7.5) $\quad \mathcal{R}\left(T^{k}\right)+\mathcal{N}\left(T^{j}\right)$ is closed for infinitely many $(k, j) \in \mathbb{Z}_{+} \times \mathbb{N}$ and there exists a strictly increasing sequence $\left(n_{k}\right)_{k \in \mathbb{N}}$ of nonnegative 
integers such that

$$
\sum_{k=0}^{\infty} \operatorname{dist}\left(x, \mathcal{N}\left(T^{n_{k}}\right)\right)<\infty \quad \text { for every } x \in \overline{\bigcup_{n \in \mathbb{N}} \mathcal{N}\left(T^{n}\right)}
$$

(4.7.6) $\quad \kappa(T)<\infty$ and there exists $N \in[0, \infty)$ such that

$$
\sum_{n=\kappa(T)}^{\infty}\left\|T^{n} x\right\|_{X} / \gamma\left(T^{n}\right) \leq N\|x\|_{X} \quad \text { for every } x \in \overline{\bigcup_{n \in \mathbb{N}} \mathcal{N}\left(T^{n}\right)}
$$

(4.7.7) $\quad \kappa(T)<\infty$ and $\sum_{n=\kappa(T)}^{\infty}\left\|T^{n} x\right\|_{X} / \gamma\left(T^{n}\right)<\infty$ for every $x \in$ $\overline{\bigcup_{n \in \mathbb{N}} \mathcal{N}\left(T^{n}\right)}$;

(4.7.8) there exists a strictly increasing sequence $\left(n_{k}\right)_{k \in \mathbb{N}}$ of nonnegative integers such that $\mathcal{R}\left(T^{n_{k}}\right)$ is closed for all $k \in \mathbb{N}$ and

$$
\sum_{k=0}^{\infty}\left\|T^{n_{k}} x\right\|_{X} / \gamma\left(T^{n_{k}}\right)<\infty \quad \text { for every } x \in \overline{\bigcup_{n \in \mathbb{N}} \mathcal{N}\left(T^{n}\right)} .
$$

Furthermore, if (4.7.1)-(4.7.8) are satisfied, we have

$$
\begin{aligned}
& \min \left\{p \in \mathbb{N}: T^{p}=0_{L(X)}\right\} \\
& =\min \left\{M \in[0, \infty): \sum_{n=0}^{\infty} \operatorname{dist}\left(x, \mathcal{N}\left(T^{n}\right)\right) \leq M\|x\|_{X}\right. \\
& \left.\quad \text { for every } x \in \overline{\bigcup_{n \in \mathbb{N}} \mathcal{N}\left(T^{n}\right)}\right\} .
\end{aligned}
$$

Proof. From Theorem 3.6, Theorem 2.3, Theorem 2.2 and (2.1) it follows that condition (4.7.1) is equivalent to each of (4.7.4)-(4.7.5) and implies (4.7.9) as well as (4.7.3).

Clearly, $(4.7 .3) \Rightarrow(4.7 .2)$. We now prove that $(4.7 .2) \Rightarrow(4.7 .1)$.

Suppose (4.7.2) is satisfied. Then, by Theorem 3.6, we have $\alpha(T) \leq$ $M<\infty$. Since $\alpha(T) \in \mathbb{N}$, we conclude that $\alpha(T) \leq[M]$ and consequently $\alpha(T)<[M]+1$. Now Theorems 2.2 and 2.3 yield (4.7.1).

We have thus proved that conditions (4.7.1)-(4.7.5) are equivalent. From Theorem 4.3 it follows that $(4.7 .1) \Rightarrow(4.7 .6)$. Clearly, $(4.7 .6) \Rightarrow(4.7 .7)$ and $(4.7 .7) \Rightarrow(4.7 .8)$. Finally, $(4.7 .8) \Rightarrow(4.7 .1)$ by Corollary 3.7 and Theorem 2.2. The proof is now complete.

5. Examples. We begin with the following property of scalar products; we give the proof for the reader's convenience.

We will denote the scalar product in any pre-Hilbert space by $\langle\cdot, \cdot\rangle$.

Lemma 5.1. Let $H$ be a pre-Hilbert space, and let $x, y \in H$ satisfy $\|x\|_{H}=\|y\|_{H}$. Then $x=y$ if and only if $\langle x, y\rangle=\|x\|_{H}^{2}$. 
Proof. Clearly, $x=y$ gives $\langle x, y\rangle=\|x\|_{H}^{2}$. Conversely, if $\langle x, y\rangle=\|x\|_{H}^{2}$, we also have $\langle y, x\rangle=\|x\|_{H}^{2}$. Since $\|x\|_{H}=\|y\|_{H}$, we conclude that

$$
\|x-y\|_{H}^{2}=\langle x-y, x-y\rangle=\|x\|_{H}^{2}-\langle x, y\rangle-\langle y, x\rangle+\|y\|_{H}^{2}=0,
$$

which establishes the desired equality $x=y$.

The following result will be used in what follows.

Lemma 5.2. Let $X$ be a Hilbert space and $\left(x_{n, k}\right)_{(n, k) \in \mathbb{N} \times \mathbb{N}}$ be an orthogonal double sequence in $X$ (that is, $\left\langle x_{n, k}, x_{m, j}\right\rangle=0$ for $\left.(n, k) \neq(m, j)\right)$. If $\sum_{n=0}^{\infty} \sum_{k=0}^{\infty}\left\|x_{n, k}\right\|_{X}^{2}<\infty$, then:

(5.2.1) $\quad \sum_{j=0}^{\infty} x_{n, j}$ and $\sum_{j=0}^{\infty} x_{j, k}$ converge in $X$ for all $n, k \in \mathbb{N}$;

(5.2.2) both $\sum_{n=0}^{\infty} \sum_{k=0}^{\infty} x_{n, k}$ and $\sum_{k=0}^{\infty} \sum_{n=0}^{\infty} x_{n, k}$ converge in $X$ and

$$
\sum_{n=0}^{\infty} \sum_{k=0}^{\infty} x_{n, k}=\sum_{k=0}^{\infty} \sum_{n=0}^{\infty} x_{n, k}
$$

$$
\left\|\sum_{n=0}^{\infty} \sum_{k=0}^{\infty} x_{n, k}\right\|_{X}^{2}=\sum_{n=0}^{\infty} \sum_{k=0}^{\infty}\left\|x_{n, k}\right\|_{X}^{2}=\sum_{k=0}^{\infty} \sum_{n=0}^{\infty}\left\|x_{n, k}\right\|_{X}^{2} .
$$

Proof. For each $n \in \mathbb{N},\left(x_{n, j}\right)_{j \in \mathbb{N}}$ is an orthogonal sequence in $X$ and $\sum_{j=0}^{\infty}\left\|x_{n, j}\right\|_{X}^{2}<\infty$. Hence $\sum_{j=0}^{\infty} x_{n, j}$ converges in $X$. From the Fubini theorem we obtain

$$
\sum_{k=0}^{\infty} \sum_{n=0}^{\infty}\left\|x_{n, k}\right\|_{X}^{2}=\sum_{n=0}^{\infty} \sum_{k=0}^{\infty}\left\|x_{n, k}\right\|_{X}^{2}<\infty .
$$

Now the same argument as above yields convergence in $X$ of $\sum_{j=0}^{\infty} x_{j, k}$ for each $k \in \mathbb{N}$. (5.2.1) is thus proved. Notice also that

$$
\begin{aligned}
\left\|\sum_{j=0}^{\infty} x_{n, j}\right\|_{X}^{2} & =\sum_{j=0}^{\infty}\left\|x_{n, j}\right\|_{X}^{2}, \\
\left\|\sum_{j=0}^{\infty} x_{j, k}\right\|_{X}^{2} & =\sum_{j=0}^{\infty}\left\|x_{j, k}\right\|_{X}^{2} \quad \text { for all } n, k \in \mathbb{N} .
\end{aligned}
$$

Now we prove (5.2.2) and (5.2.3). We begin by remarking that the sequence $\left(\sum_{k=0}^{\infty} x_{n, k}\right)_{n \in \mathbb{N}}$ is orthogonal in $X$ : indeed, if $n, m \in \mathbb{N}$ satisfy $n \neq m$, we have

$$
\left\langle\sum_{k=0}^{\infty} x_{n, k}, \sum_{j=0}^{\infty} x_{m, j}\right\rangle=\sum_{k=0}^{\infty} \sum_{j=0}^{\infty}\left\langle x_{n, k}, x_{m, j}\right\rangle=0 .
$$

From (5.2.4) and (5.2.5) we obtain

$$
\sum_{n=0}^{\infty}\left\|\sum_{k=0}^{\infty} x_{n, k}\right\|_{X}^{2}=\sum_{n=0}^{\infty} \sum_{k=0}^{\infty}\left\|x_{n, k}\right\|_{X}^{2}<\infty
$$


from which we conclude that $\sum_{n=0}^{\infty} \sum_{k=0}^{\infty} x_{n, k}$ converges in $X$ and

$$
\left\|\sum_{n=0}^{\infty} \sum_{k=0}^{\infty} x_{n, k}\right\|_{X}^{2}=\sum_{n=0}^{\infty} \sum_{k=0}^{\infty}\left\|x_{n, k}\right\|_{X}^{2} .
$$

Notice that (5.2.6), together with (5.2.4), gives (5.2.3).

By applying the same argument as above, from (5.2.4) we derive that $\sum_{k=0}^{\infty} \sum_{n=0}^{\infty} x_{n, k}$ converges in $X$ and

$$
\left\|\sum_{k=0}^{\infty} \sum_{n=0}^{\infty} x_{n, k}\right\|_{X}^{2}=\sum_{k=0}^{\infty} \sum_{n=0}^{\infty}\left\|x_{n, k}\right\|_{X}^{2} .
$$

Now (5.2.4), (5.2.6) and (5.2.7) yield the equality

$$
\left\|\sum_{n=0}^{\infty} \sum_{k=0}^{\infty} x_{n, k}\right\|_{X}=\left\|\sum_{k=0}^{\infty} \sum_{n=0}^{\infty} x_{n, k}\right\|_{X} .
$$

It remains to prove that $\sum_{n=0}^{\infty} \sum_{k=0}^{\infty} x_{n, k}=\sum_{k=0}^{\infty} \sum_{n=0}^{\infty} x_{n, k}$. For each $p, q \in \mathbb{N}$, let $\delta_{p q}$ denote the Kronecker symbol. Since

$$
\begin{aligned}
& \left\langle\sum_{n=0}^{\infty} \sum_{k=0}^{\infty} x_{n, k}, \sum_{k=0}^{\infty} \sum_{n=0}^{\infty} x_{n, k}\right\rangle=\sum_{n=0}^{\infty} \sum_{k=0}^{\infty} \sum_{j=0}^{\infty} \sum_{h=0}^{\infty}\left\langle x_{n, k}, x_{h, j}\right\rangle \\
& \quad=\sum_{n=0}^{\infty} \sum_{k=0}^{\infty} \sum_{j=0}^{\infty} \sum_{h=0}^{\infty} \delta_{n h} \delta_{k j}\left\|x_{n, k}\right\|_{X}^{2}=\sum_{n=0}^{\infty} \sum_{k=0}^{\infty}\left\|x_{n, k}\right\|_{X}^{2}=\left\|\sum_{n=0}^{\infty} \sum_{k=0}^{\infty} x_{n, k}\right\|_{X}^{2}
\end{aligned}
$$

by (5.2.6), from (5.2.8) and Lemma 5.1 we obtain the desired result.

For every subset $S$ of a linear space $V$, let $\operatorname{Span}(S)$ denote the linear subspace of $V$ spanned by $S$.

We will also need the following property of Hilbert spaces. For the reader's convenience, we include a proof.

LEMMA 5.3. Let $X$ be a Hilbert space and let $\left(X_{n}\right)_{n \in \mathbb{N}}$ be a sequence of pairwise orthogonal closed subspaces of $X$. For each $n \in \mathbb{N}$, let $P_{n}$ denote the orthogonal projection of $X$ onto $X_{n}$. If $\operatorname{Span}\left(\bigcup_{n \in \mathbb{N}} X_{n}\right)$ is dense in $X$, then for each $x \in X$ we have

$$
x=\sum_{n=0}^{\infty} P_{n} x, \quad\|x\|_{X}^{2}=\sum_{n=0}^{\infty}\left\|P_{n} x\right\|_{X}^{2} .
$$

Proof. Indeed, for each $n \in \mathbb{N}, \sum_{k=0}^{n} P_{k}$ is the orthogonal projection of $X$ onto the closed subspace $\bigoplus_{k=0}^{n} X_{k}=\operatorname{Span}\left(\bigcup_{k=0}^{n} X_{k}\right)$. Hence, for each $x \in X$, the norm $\left\|x-\sum_{k=0}^{n} P_{k} x\right\|_{X}$ coincides with the distance from $x$ to $\operatorname{Span}\left(\bigcup_{k=0}^{n} X_{k}\right)$. Since $\operatorname{Span}\left(\bigcup_{k \in \mathbb{N}} X_{k}\right)$ is dense in $X$, we conclude that $\lim _{n \rightarrow \infty}\left\|x-\sum_{k=0}^{n} P_{k} x\right\|_{X}=0$ for each $x \in X$. Since $\left\|\sum_{k=0}^{n} P_{k} x\right\|_{X}^{2}=$ $\sum_{k=0}^{n}\left\|P_{k} x\right\|_{X}^{2}$ for each $n \in \mathbb{N}$ and $x \in X$, we get the desired result. 
The following question was posed in the final comments of [MZ].

QUESTION. Is every quasinilpotent bounded linear operator $T$ on a Banach space $X$, such that $\mathcal{R}\left(T^{n}\right)$ is closed for each $n \in \mathbb{N}$, nilpotent?

As explained in the Introduction, the operator constructed in the proof of [Ap, Theorem 3], in the special case $\sigma=\{0\}$, provides a negative answer to the question above. In the following example, we construct another nonnilpotent quasinilpotent bounded linear operator on an infinite-dimensional Hilbert space, all the iterates of which have closed range. We also exactly compute the reduced minimum moduli of the iterates of our operator, besides giving a formula to determine their norms.

For each Hilbert space $X$, let $\operatorname{dim}_{\mathrm{H}}(X)$ denote the Hilbert dimension of $X$.

EXAmple 5.4. Let $X$ be an infinite-dimensional Hilbert space. Then there exists a sequence $\left(X_{n}\right)_{n \in \mathbb{N}}$ of pairwise orthogonal closed subspaces of $X$ satisfying $\operatorname{dim}_{\mathrm{H}}\left(X_{n}\right)=\operatorname{dim}_{\mathrm{H}}(X)$ for every $n \in \mathbb{N}$ and such that $\operatorname{Span}\left(\bigcup_{n \in \mathbb{N}} X_{n}\right)$ is dense in $X$. Furthermore, for each $n \in \mathbb{N}$, there exists a sequence $\left(X_{n, k}\right)_{k \in \mathbb{N}}$ of pairwise orthogonal closed subspaces of $X_{n}$ satisfying $\operatorname{dim}_{\mathrm{H}}\left(X_{n, k}\right)=\operatorname{dim}_{\mathrm{H}}\left(X_{n}\right)=\operatorname{dim}_{\mathrm{H}}(X)$ for all $k \in \mathbb{N}$. Hence $X_{n, k} \perp X_{m, h}$ for all $(n, k),(m, h) \in \mathbb{N} \times \mathbb{N}$ such that $(n, k) \neq(m, h)$.

For each $n \in \mathbb{Z}_{+}$and $j \in\{0, \ldots, n-1\}$, let $U_{n, j}: X_{n} \rightarrow X_{j, n-1-j}$ be a linear isometry. We remark that

$$
\begin{gathered}
\mathcal{R}\left(U_{n, j}\right) \perp \mathcal{R}\left(U_{m, k}\right) \quad \text { for all } n, m \in \mathbb{Z}_{+}, j \in\{0, \ldots, n-1\} \text { and } \\
k \in\{0, \ldots, m-1\} \text { satisfying }(n, j) \neq(m, k)
\end{gathered}
$$

(as $\mathcal{R}\left(U_{n, j}\right) \subset X_{j, n-1-j}, \mathcal{R}\left(U_{m, k}\right) \subset X_{k, m-1-k}$ and $(j, n-1-j) \neq(k, m-$ $1-k)$ ). For each $n \in \mathbb{N}$, let $P_{n}$ denote the orthogonal projection of $X$ onto $X_{n}$. From Lemma 5.3 it follows that for each $x \in X$,

$$
x=\sum_{n=0}^{\infty} P_{n} x, \quad\|x\|_{X}^{2}=\sum_{n=0}^{\infty}\left\|P_{n} x\right\|_{X}^{2} .
$$

We fix a sequence $\left(\varepsilon_{n}\right)_{n \in \mathbb{N}}$ of strictly positive numbers such that $\sum_{n=0}^{\infty} \varepsilon_{n}^{2}$ $<\infty$. Now let $x \in X$. From (5.4.2) we obtain

$$
\sum_{j=0}^{\infty} \varepsilon_{j}^{2} \sum_{n=j+1}^{\infty}\left\|U_{n, j} P_{n} x\right\|_{X}^{2}=\sum_{j=0}^{\infty} \varepsilon_{j}^{2} \sum_{n=j+1}^{\infty}\left\|P_{n} x\right\|_{X}^{2} \leq\|x\|_{X}^{2} \sum_{j=0}^{\infty} \varepsilon_{j}^{2} .
$$

Since, by (5.4.1), we have $\left\langle U_{n, j} P_{n} x, U_{m, k} P_{m} x\right\rangle=0$ for all $n, m \in \mathbb{Z}_{+}, j \in$ $\{0, \ldots, n-1\}$ and $k \in\{0, \ldots, m-1\}$ satisfying $(n, j) \neq(m, k)$, from Lemma 5.2 we conclude that the series $\sum_{n=j+1}^{\infty} U_{n, j} P_{n} x$ converges in $X$ for each $j \in \mathbb{N}$, both $\sum_{j=0}^{\infty} \varepsilon_{j} \sum_{n=j+1}^{\infty} U_{n, j} P_{n} x$ and $\sum_{n=1}^{\infty} \sum_{j=0}^{n-1} \varepsilon_{j} U_{n, j} P_{n} x$ converge 
in $X$ and

$$
\sum_{n=1}^{\infty} \sum_{j=0}^{n-1} \varepsilon_{j} U_{n, j} P_{n} x=\sum_{j=0}^{\infty} \varepsilon_{j} \sum_{n=j+1}^{\infty} U_{n, j} P_{n} x .
$$

Lemma 5.2, together with (5.4.3), also yields

$$
\left\|\sum_{n=1}^{\infty} \sum_{j=0}^{n-1} \varepsilon_{j} U_{n, j} P_{n} x\right\|_{X} \leq\|x\|_{X} \sqrt{\sum_{n=0}^{\infty} \varepsilon_{n}^{2}} .
$$

Consider the linear operator $T: X \rightarrow X$ defined by

$$
T x=\sum_{n=1}^{\infty} \sum_{j=0}^{n-1} \varepsilon_{j} U_{n, j} P_{n} x \quad \text { for each } x \in X .
$$

From (5.4.5) it follows that $T \in L(X)$ and $\|T\|_{L(X)} \leq \sqrt{\sum_{n=0}^{\infty} \varepsilon_{n}^{2}}$.

Since $\mathcal{R}\left(U_{n, j}\right) \subset X_{j, n-1-j} \subset X_{j}$ for each $j \in \mathbb{N}$ and $n \geq j+1$, from (5.4.4) it follows that

$$
P_{j} T x=\varepsilon_{j} \sum_{n=j+1}^{\infty} U_{n, j} P_{n} x \quad \text { for all } j \in \mathbb{N}, x \in X .
$$

Hence, by (5.4.1), we have

$$
\left\|P_{j} T x\right\|_{X}^{2}=\varepsilon_{j}^{2} \sum_{n=j+1}^{\infty}\left\|U_{n, j} P_{n} x\right\|_{X}^{2}=\varepsilon_{j}^{2} \sum_{n=j+1}^{\infty}\left\|P_{n} x\right\|_{X}^{2}
$$

for all $j \in \mathbb{N}, x \in X$. Now let $\left(\left(a_{k, n}\right)_{n \geq k}\right)_{k \in \mathbb{N}}$ be the sequence of sequences of real numbers which is defined by induction as follows:

$$
a_{0, n}=1 \quad \text { for every } n \in \mathbb{N}
$$

and, for each $k \in \mathbb{N}$,

$$
a_{k+1, n}=\sum_{j=k}^{n-1} \varepsilon_{j}^{2} a_{k, j} \quad \text { for every } n \geq k+1 .
$$

We prove that, for each $k \in \mathbb{N}$, we have

$$
\left\|T^{k} x\right\|_{X}^{2}=\sum_{n=k}^{\infty} a_{k, n}\left\|P_{n} x\right\|_{X}^{2} \quad \text { for all } x \in X .
$$

We proceed by induction. From (5.4.2) it follows that (5.4.7) holds for $k=0$. Now suppose (5.4.7) is satisfied for some $k \in \mathbb{N}$, and let $x \in X$. By using 
(5.4.6), we obtain

$$
\begin{aligned}
\left\|T^{k+1} x\right\|_{X}^{2} & =\left\|T^{k}(T x)\right\|_{X}^{2}=\sum_{j=k}^{\infty} a_{k, j}\left\|P_{j} T x\right\|_{X}^{2}=\sum_{j=k}^{\infty} a_{k, j} \varepsilon_{j}^{2} \sum_{n=j+1}^{\infty}\left\|P_{n} x\right\|_{X}^{2} \\
& =\sum_{n=k+1}^{\infty}\left(\sum_{j=k}^{n-1} \varepsilon_{j}^{2} a_{k, j}\right)\left\|P_{n} x\right\|_{X}^{2}=\sum_{n=k+1}^{\infty} a_{k+1, n}\left\|P_{n} x\right\|_{X}^{2},
\end{aligned}
$$

which establishes the desired equality for $k+1$. Hence (5.4.7) holds for each $k \in \mathbb{N}$.

Proceeding by induction on $k$, it is not difficult to check that

$$
a_{k, n}>0 \quad \text { for every } k \in \mathbb{N} \text { and } n \geq k
$$

and that the sequence $\left(a_{k, n}\right)_{n \geq k}$ is bounded for every $k \in \mathbb{N}$ (as $\sum_{j=0}^{\infty} \varepsilon_{j}^{2}$ $<\infty)$. We also remark that $\left(a_{k, n}\right)_{n \geq k}$ is nondecreasing for each $k \in \mathbb{N}$; indeed, by (5.4.8), it is strictly increasing for each $k \in \mathbb{Z}_{+}$. Then, if we set

$$
a_{k}=\sup \left\{a_{k, n}: n \in \mathbb{N}, n \geq k\right\} \quad \text { for each } k \in \mathbb{N},
$$

we have $a_{k} \in(0, \infty)$ for all $k \in \mathbb{N}$. Furthermore, from (5.4.7) we conclude that

$$
a_{k, k} \sum_{n=k}^{\infty}\left\|P_{n} x\right\|_{X}^{2} \leq\left\|T^{k} x\right\|_{X}^{2} \leq a_{k} \sum_{n=k}^{\infty}\left\|P_{n} x\right\|_{X}^{2}
$$

for all $x \in X, k \in \mathbb{N}$. Since $a_{k, k}>0$ for all $k \in \mathbb{N}$ by (5.4.8), from (5.4.2) and (5.4.9) we derive that, for each $k \in \mathbb{Z}_{+}$and $x \in X$, we have

$$
x \in \mathcal{N}\left(T^{k}\right) \Leftrightarrow \sum_{n=k}^{\infty}\left\|P_{n} x\right\|_{X}^{2}=0 \Leftrightarrow x=\sum_{j=0}^{k-1} P_{j} x \Leftrightarrow x \in \bigoplus_{j=0}^{k-1} X_{j} .
$$

Hence

$$
\mathcal{N}\left(T^{k}\right)=\bigoplus_{j=0}^{k-1} X_{j} \subsetneq X \quad \text { for all } k \in \mathbb{Z}_{+},
$$

and consequently $T$ is not nilpotent.

We now prove that $\mathcal{R}\left(T^{k}\right)$ is closed for all $k \in \mathbb{N}$. Let $k \in \mathbb{Z}_{+}$. Since $\sum_{j=0}^{k-1} P_{j}$ is the orthogonal projection of $X$ onto $\mathcal{N}\left(T^{k}\right)$ by (5.4.10), from (5.4.9) we conclude that

$$
\left\|T^{k} x\right\|_{X} \geq \sqrt{a_{k, k}} \operatorname{dist}\left(x, \mathcal{N}\left(T^{k}\right)\right) \quad \text { for every } x \in X .
$$

Hence $\gamma\left(T^{k}\right) \geq \sqrt{a_{k, k}}>0$, and consequently $\mathcal{R}\left(T^{k}\right)$ is closed by Theorem 2.4 .

Although the inequality above is sufficient in order to get closedness of $\mathcal{R}\left(T^{k}\right)$, we now determine the exact value of $\gamma\left(T^{k}\right)$, which will be needed in the next example. 
We remark that $X_{k} \subset \mathcal{N}\left(T^{k}\right)^{\perp}$ by (5.4.10). This, together with (5.4.7), yields

$$
\sqrt{a_{k, k}}\|x\|_{X}=\left\|T^{k} x\right\|_{X} \geq \gamma\left(T^{k}\right)\|x\|_{X} \quad \text { for all } x \in X_{k},
$$

from which, since $X_{k}$ is nonzero, we derive that $\gamma\left(T^{k}\right) \leq \sqrt{a_{k, k}}$. Hence $\gamma\left(T^{k}\right)=\sqrt{a_{k, k}}$.

Since $\sqrt{a_{0,0}}=1$ and $\sqrt{a_{h, h}}=\varepsilon_{h-1} \sqrt{a_{h-1, h-1}}$ for every $h \in \mathbb{Z}_{+}$, we conclude that

$$
\gamma\left(T^{k}\right)=\prod_{j=0}^{k-1} \varepsilon_{j} \quad \text { for each } k \in \mathbb{Z}_{+} .
$$

Finally, we prove that $T$ is quasinilpotent. Let $k \in \mathbb{N}$. From (5.4.2) and (5.4.9) it follows that $\left\|T^{k}\right\|_{L(X)} \leq \sqrt{a_{k}}$. On the other hand, if we choose a sequence $\left(x_{n}\right)_{n \geq k}$ of norm one elements of $X$ satisfying $x_{n} \in X_{n}$ for every $n \geq k$, from (5.4.7) we obtain

$$
\begin{aligned}
\left\|T^{k}\right\|_{L(X)} & \geq \sup \left\{\left\|T^{k} x_{n}\right\|_{X}: n \in \mathbb{N}, n \geq k\right\} \\
& =\sup \left\{\sqrt{a_{k, n}}: n \in \mathbb{N}, n \geq k\right\}=\sqrt{a_{k}} .
\end{aligned}
$$

Hence $\left\|T^{k}\right\|_{L(X)}=\sqrt{a_{k}}$.

We remark that $a_{0}=1$. Since the sequence $\left(a_{m, n}\right)_{n \geq m}$ is nondecreasing for each $m \in \mathbb{N}$, we also have

$$
\begin{aligned}
a_{h} & =\lim _{n \rightarrow \infty} a_{h, n}=\lim _{n \rightarrow \infty} \sum_{j=h-1}^{n-1} \varepsilon_{j}^{2} a_{h-1, j} \\
& =\sum_{j=h-1}^{\infty} \varepsilon_{j}^{2} a_{h-1, j} \leq a_{h-1} \sum_{j=h-1}^{\infty} \varepsilon_{j}^{2}
\end{aligned}
$$

for all $h \in \mathbb{Z}_{+}$. Proceeding by induction, it is not difficult to verify that

$$
a_{k} \leq \prod_{j=0}^{k-1}\left(\sum_{n=j}^{\infty} \varepsilon_{n}^{2}\right) \quad \text { for every } k \in \mathbb{Z}_{+} .
$$

Actually, the inequality in (5.4.13) can be replaced by equality for $k=1$ (as $a_{0, n}=1$ for all $n \in \mathbb{N}$ ), whereas it is strict for $k \geq 2$ (as the sequence $\left(a_{k-1, n}\right)_{n \geq k-1}$ is strictly increasing).

Since $\left\|T^{k}\right\|_{L(X)}=\sqrt{a_{k}}$ for each $k \in \mathbb{N}$, from (5.4.13) we obtain

$$
\left\|T^{k}\right\|_{L(X)} \leq \sqrt{\prod_{j=0}^{k-1}\left(\sum_{n=j}^{\infty} \varepsilon_{n}^{2}\right)} \quad \text { for every } k \in \mathbb{Z}_{+} .
$$


Since $\sum_{n=0}^{\infty} \varepsilon_{n}^{2}<\infty$, we have $\lim _{j \rightarrow \infty} \sum_{n=j}^{\infty} \varepsilon_{n}^{2}=0$. Then

$$
\sqrt[k]{\left\|T^{k}\right\|_{L(X)}} \leq \sqrt{\prod_{j=0}^{k-1}\left(\sum_{n=j}^{\infty} \varepsilon_{n}^{2}\right)^{1 / k}} \underset{k \rightarrow \infty}{\longrightarrow} 0
$$

from which we conclude that $\lim _{k \rightarrow \infty} \sqrt[k]{\left\|T^{k}\right\|_{L(X)}}=0$. Hence $T$ is quasinilpotent.

We remark that from Theorem 2.5 it follows that, if $X$ is a complex Hilbert space, then $\mathfrak{p}(T)$ has closed range for every polynomial $\mathfrak{p}$ with complex coefficients.

If $X$ is a real Hilbert space, Theorem 2.5 yields closedness of $\mathcal{R}(\mathfrak{p}(\widetilde{T}))$ for every polynomial $\mathfrak{p}$ with complex coefficients. Since $\mathfrak{q}(\widetilde{T})=\widetilde{\mathfrak{q}(T)}$ for every polynomial $\mathfrak{q}$ with real coefficients, it follows that $\mathfrak{q}(T)$ has closed range for every polynomial $\mathfrak{q}$ with real coefficients.

The following example shows that the equivalent conditions (4.5.1) and (4.5.2) are not sufficient in order that $T$ be nilpotent.

ExAMPLE 5.5 Let $X$ be an infinite-dimensional Hilbert space, and let $T \in L(X)$ be the operator introduced in Example 5.4 (to which we refer for all the definitions and notations we do not recall here). We recall that $T$ is quasinilpotent and $\kappa(T)=1$. Also, since $\mathcal{N}\left(T^{k}\right)=\bigoplus_{j=0}^{k-1} X_{j}$ for every $k \in \mathbb{Z}_{+}(\operatorname{see}(5.4 .10))$ and $\operatorname{Span}\left(\bigcup_{n \in \mathbb{N}} X_{n}\right)$ is dense in $X$, it follows that $\bigcup_{n \in \mathbb{N}} \mathcal{N}\left(T^{n}\right)$ is dense in $X$. However, $T$ need not satisfy (4.5.1) and (4.5.2), as the sequence $\left(\left\|T^{n}\right\|_{L(X)} / \gamma\left(T^{n}\right)\right)_{n \in \mathbb{N}}$ need not be bounded. Indeed, since the sequence $\left(a_{m, n}\right)_{n \geq m}$ (introduced in Example 5.4) is nondecreasing for each $m \in \mathbb{N}$, by $(5.4 .12)$ we have

$$
a_{k}=\sum_{j=k-1}^{\infty} \varepsilon_{j}^{2} a_{k-1, j} \geq a_{k-1, k-1} \sum_{j=k-1}^{\infty} \varepsilon_{j}^{2} \quad \text { for every } k \in \mathbb{Z}_{+} .
$$

Since $\left\|T^{k}\right\|_{L(X)}=\sqrt{a_{k}}$ and $\gamma\left(T^{k}\right)=\sqrt{a_{k, k}}=\varepsilon_{k-1} \sqrt{a_{k-1, k-1}}$ for each $k \in \mathbb{Z}_{+}$(see Example 5.4), from (5.5.1) we conclude that, for each $k \in \mathbb{Z}_{+}$, we have

$$
\begin{aligned}
\frac{\left\|T^{k}\right\|_{L(X)}}{\gamma\left(T^{k}\right)} & =\frac{\sqrt{a_{k}}}{\varepsilon_{k-1} \sqrt{a_{k-1, k-1}}} \\
& \geq \frac{\sqrt{a_{k-1, k-1} \sum_{j=k-1}^{\infty} \varepsilon_{j}^{2}}}{\varepsilon_{k-1} \sqrt{a_{k-1, k-1}}}=\frac{1}{\varepsilon_{k-1}} \sqrt{\sum_{j=k-1}^{\infty} \varepsilon_{j}^{2} .}
\end{aligned}
$$

Then, if for instance we take

$$
\varepsilon_{j}=\frac{1}{\sqrt{(j+1)(j+2)}} \quad \text { for every } j \in \mathbb{N}
$$


from (5.5.2) we obtain

$$
\begin{aligned}
& \frac{\left\|T^{k}\right\|_{L(X)}}{\gamma\left(T^{k}\right)} \geq \sqrt{k(k+1)} \sqrt{\sum_{j=k-1}^{\infty} \frac{1}{(j+1)(j+2)}} \\
= & \sqrt{k(k+1) \sum_{j=k-1}^{\infty}\left(\frac{1}{(j+1)}-\frac{1}{(j+2)}\right)}=\sqrt{k(k+1) \frac{1}{k}} \\
= & \sqrt{k+1} \underset{k \rightarrow \infty}{\longrightarrow} \infty .
\end{aligned}
$$

Anyway, the sequence $\left(\varepsilon_{n}\right)_{n \in \mathbb{N}}$ can also be chosen so that the sequence $\left(\left\|T^{n}\right\|_{L(X)} / \gamma\left(T^{n}\right)\right)_{n \in \mathbb{N}}$ is bounded. Indeed, from (5.4.11) and (5.4.14) we derive that, for each $k \in \mathbb{Z}_{+}$, we have

$$
\begin{aligned}
\frac{\left\|T^{k}\right\|_{L(X)}}{\gamma\left(T^{k}\right)} & \leq \frac{\sqrt{\prod_{j=0}^{k-1}\left(\sum_{n=j}^{\infty} \varepsilon_{n}^{2}\right)}}{\prod_{j=0}^{k-1} \varepsilon_{j}}=\sqrt{\prod_{j=0}^{k-1}\left(\frac{1}{\varepsilon_{j}^{2}} \sum_{n=j}^{\infty} \varepsilon_{n}^{2}\right)} \\
& =\exp \left(\frac{1}{2} \sum_{j=0}^{k-1} \log \left(1+\sum_{n=j+1}^{\infty} \frac{\varepsilon_{n}^{2}}{\varepsilon_{j}^{2}}\right)\right)
\end{aligned}
$$

Hence, if

$$
\sum_{j=0}^{\infty} \log \left(1+\sum_{n=j+1}^{\infty} \frac{\varepsilon_{n}^{2}}{\varepsilon_{j}^{2}}\right)<\infty,
$$

then by (5.5.3) we have

$$
\frac{\left\|T^{k}\right\|_{L(X)}}{\gamma\left(T^{k}\right)} \leq \exp \left(\frac{1}{2} \sum_{j=0}^{\infty} \log \left(1+\sum_{n=j+1}^{\infty} \frac{\varepsilon_{n}^{2}}{\varepsilon_{j}^{2}}\right)\right) \quad \text { for all } k \in \mathbb{N} .
$$

Notice the inequality above is satisfied for $k=0$ as well, since

$$
\frac{\left\|T^{0}\right\|_{L(X)}}{\gamma\left(T^{0}\right)}=\frac{\left\|I_{X}\right\|_{L(X)}}{\gamma\left(I_{X}\right)}=1, \quad \sum_{j=0}^{\infty} \log \left(1+\sum_{n=j+1}^{\infty} \frac{\varepsilon_{n}^{2}}{\varepsilon_{j}^{2}}\right)>0 .
$$

Now we show that $\left(\varepsilon_{n}\right)_{n \in \mathbb{N}}$ can be chosen so that (5.5.4) be satisfied. If for instance we set

$$
\varepsilon_{n}=\frac{1}{(n+1) !} \quad \text { for every } n \in \mathbb{N}
$$

we have

$$
\sum_{n=j+1}^{\infty} \frac{\varepsilon_{n}^{2}}{\varepsilon_{j}^{2}}=\sum_{n=j+1}^{\infty}\left(\frac{(j+1) !}{(n+1) !}\right)^{2}=\sum_{n=j+1}^{\infty}\left(\prod_{h=j+2}^{n+1} \frac{1}{h^{2}}\right)
$$




$$
\begin{aligned}
& \leq \sum_{n=j+1}^{\infty}\left(\frac{1}{j+2}\right)^{2(n-j)}=\sum_{n=1}^{\infty}\left(\frac{1}{j+2}\right)^{2 n} \\
& =\frac{\frac{1}{(j+2)^{2}}}{1-\frac{1}{(j+2)^{2}}}=\frac{1}{j^{2}+4 j+3}
\end{aligned}
$$

for each $j \in \mathbb{N}$, and consequently

$$
\begin{aligned}
\sum_{j=0}^{\infty} \log \left(1+\sum_{n=j+1}^{\infty} \frac{\varepsilon_{n}^{2}}{\varepsilon_{j}^{2}}\right) & \leq \sum_{j=0}^{\infty} \log \left(1+\frac{1}{j^{2}+4 j+3}\right) \\
& \leq \sum_{j=0}^{\infty} \frac{1}{j^{2}+4 j+3}<+\infty
\end{aligned}
$$

which gives (5.5.4). Hence, by (5.5.5), the sequence $\left(\left\|T^{k}\right\|_{L(X)} / \gamma\left(T^{k}\right)\right)_{k \in \mathbb{N}}$ is bounded. Therefore, with the latter choice of $\left(\varepsilon_{n}\right)_{n \in \mathbb{N}}$, the operator $T$ satisfies the equivalent conditions (4.5.1) and (4.5.2). Yet, as we have proved in Example 5.4, $T$ is not nilpotent.

REMARK 5.6. We now prove that the operator $T$ in Examples 5.4 and 5.5 leaves open the question whether (4.4.1) is sufficient for nilpotency, because for no choice of the sequence $\left(\varepsilon_{n}\right)_{n \in \mathbb{N}}$ does the series $\sum_{n=1}^{\infty} T^{n} x / \gamma\left(T^{n}\right)$ converge in $X$ for every $x \in X$.

Indeed, let $X$ be an infinite-dimensional Hilbert space, and let $T \in L(X)$ be the operator defined in Example 5.4. We begin by remarking that, since (as proved in Example 5.4)

$$
P_{j} T x=\varepsilon_{j} \sum_{n=j+1}^{\infty} U_{n, j} P_{n} x \quad \text { for all } j \in \mathbb{N} \text { and } x \in X,
$$

we have $T x \in \bigoplus_{k=0}^{n-1} X_{k}$ for every $x \in \bigoplus_{k=0}^{n} X_{k}$ and $n \in \mathbb{Z}_{+}$. Hence

$$
T^{k} x \in \bigoplus_{j=0}^{n-k} X_{j} \quad \text { for every } x \in \bigoplus_{j=0}^{n} X_{j}, k \in\{0, \ldots, n\}, n \in \mathbb{Z}_{+} .
$$

Now we prove that, for each $k \in \mathbb{N}$, we have

$$
\begin{aligned}
& \left\langle P_{n} T^{k} x, P_{n} T^{k} y\right\rangle=0 \text { for all } n \in \mathbb{N} \text { and } x, y \in X \\
& \text { satisfying }\left\langle P_{j} x, P_{j} y\right\rangle=0 \text { for all } j \in \mathbb{N} .
\end{aligned}
$$

We proceed by induction on $k$. Clearly, (5.6.3) is satisfied for $k=0$. Now suppose (5.6.3) is satisfied for some $k \in \mathbb{N}$, and let $x, y \in X$ be such that $\left\langle P_{n} x, P_{n} y\right\rangle=0$ for every $n \in \mathbb{N}$. Then, for each $n \in \mathbb{N}$, since the $U_{m, n}$, $m \geq n+1$, are isometries, and moreover $\mathcal{R}\left(U_{m, n}\right) \perp \mathcal{R}\left(U_{j, n}\right)$ for $m \neq j$ by 
(5.4.1), from (5.6.1) we obtain

$$
\begin{gathered}
\left\langle P_{n} T x, P_{n} T y\right\rangle=\left\langle\varepsilon_{n} \sum_{m=n+1}^{\infty} U_{m, n} P_{m} x, \varepsilon_{n} \sum_{j=n+1}^{\infty} U_{j, n} P_{j} y\right\rangle \\
=\varepsilon_{n}^{2} \sum_{m=n+1}^{\infty} \sum_{j=n+1}^{\infty}\left\langle U_{m, n} P_{m} x, U_{j, n} P_{j} y\right\rangle \\
=\varepsilon_{n}^{2} \sum_{m=n+1}^{\infty}\left\langle U_{m, n} P_{m} x, U_{m, n} P_{m} y\right\rangle=\varepsilon_{n}^{2} \sum_{m=n+1}^{\infty}\left\langle P_{m} x, P_{m} y\right\rangle=0 .
\end{gathered}
$$

Since (5.6.3) is satisfied for $k$, we conclude that

$$
0=\left\langle P_{n} T^{k}(T x), P_{n} T^{k}(T y)\right\rangle=\left\langle P_{n} T^{k+1} x, P_{n} T^{k+1} y\right\rangle \quad \text { for all } n \in \mathbb{N} .
$$

Hence (5.6.3) is satisfied for $k+1$, which gives the desired result.

Now, for each $n \in \mathbb{Z}_{+}$, let $x_{n} \in X_{n}$ be such that $\left\|x_{n}\right\|_{X}=1$ (such an $x_{n}$ exists, as $X_{n}$ is nonzero). From (5.6.2) it follows that $P_{n} T^{k} x_{n}=0_{X}$ for every $k=1, \ldots, n$. Since $x_{n}=P_{n} x_{n}$, and consequently $P_{j} x_{n}=0_{X}$ for all $j \in \mathbb{N} \backslash\{n\}$, we conclude that

$$
\left\langle P_{j} x_{n}, P_{j} T^{k} x_{n}\right\rangle=0 \quad \text { for all } j \in \mathbb{N}, k=1, \ldots, n .
$$

From (5.6.3) it follows that

$$
\left\langle P_{j} T^{h} x_{n}, P_{j} T^{h+k} x_{n}\right\rangle=0 \quad \text { for all } j, h \in \mathbb{N}, k=1, \ldots, n .
$$

Notice that $T^{n+1} x_{n}=0_{X}$, as $X_{n} \subset \bigoplus_{j=0}^{n} X_{j}=\mathcal{N}\left(T^{n+1}\right)$ (see (5.4.10)). Since, by (5.6.4), $\left\langle P_{j} T^{h} x_{n}, P_{j} T^{k} x_{n}\right\rangle=0$ for all $j \in \mathbb{N}$ and $h, k \in\{0, \ldots, n\}$ with $k \neq h$, from (5.4.2) we obtain

$$
\begin{aligned}
\left\langle T^{h} x_{n}, T^{k} x_{n}\right\rangle & =\left\langle\sum_{j=0}^{\infty} P_{j} T^{h} x_{n}, \sum_{m=0}^{\infty} P_{m} T^{k} x_{n}\right\rangle \\
& =\sum_{j=0}^{\infty} \sum_{m=0}^{\infty}\left\langle P_{j} T^{h} x_{n}, P_{m} T^{k} x_{n}\right\rangle \\
& =\sum_{j=0}^{\infty}\left\langle P_{j} T^{h} x_{n}, P_{j} T^{k} x_{n}\right\rangle=0
\end{aligned}
$$

for all $h, k \in\{0, \ldots, n\}$ satisfying $h \neq k$.

Since $\mathcal{N}\left(T^{n}\right)=\bigoplus_{k=0}^{n-1} X_{k}$ by (5.4.10), and the closed subspaces $X_{j}$, $j \in \mathbb{N}$, are pairwise orthogonal, we conclude that $x_{n} \in \mathcal{N}\left(T^{n}\right)^{\perp}$. Hence $x_{n} \in \mathcal{N}\left(T^{k}\right)^{\perp}$ for all $k=1, \ldots, n$, and consequently

$$
\operatorname{dist}\left(x_{n}, \mathcal{N}\left(T^{k}\right)\right)=\left\|x_{n}\right\|_{X}=1 \quad \text { for all } k=1, \ldots, n .
$$


Then, by (5.6.5), we have

$$
\left\|\sum_{k=1}^{n} \frac{T^{k} x_{n}}{\gamma\left(T^{k}\right)}\right\|_{X}^{2}=\sum_{k=1}^{n} \frac{\left\|T^{k} x_{n}\right\|_{X}^{2}}{\gamma\left(T^{k}\right)^{2}} \geq \sum_{k=1}^{n}\left(\operatorname{dist}\left(x_{n}, \mathcal{N}\left(T^{k}\right)\right)\right)^{2}=n .
$$

From (5.6.6) it follows that

$$
\left\|\sum_{k=1}^{n} \frac{T^{k}}{\gamma\left(T^{k}\right)}\right\|_{L(X)} \geq \sqrt{n} \quad \text { for every } n \in \mathbb{Z}_{+} .
$$

Hence the sequence $\left(\sum_{k=1}^{n} T^{k} / \gamma\left(T^{k}\right)\right)_{n \in \mathbb{Z}_{+}}$is not bounded in $L(X)$. Then, by the uniform boundedness principle, there exists $x_{0} \in X$ such that the sequence $\left(\sum_{k=1}^{n} T^{k} x_{0} / \gamma\left(T^{k}\right)\right)_{n \in \mathbb{Z}_{+}}$is not bounded in $X$, and consequently the series $\sum_{n=1}^{\infty} T^{n} x_{0} / \gamma\left(T^{n}\right)$ does not converge in $X$. Hence $T$ does not satisfy condition (4.4.1).

The following generalization of Theorem 2.5 is a consequence of Theorem 2.6.

Proposition 5.7. Let $X$ be a complex Banach space and let $T \in L(X)$ be such that $\mathcal{R}\left(\left(\lambda I_{X}-T\right)^{n}\right)$ is closed for every $n \in \mathbb{N}$ and every $\lambda \in \sigma(T)$. Then $\mathcal{R}(f(T))$ is closed for every $f \in \mathcal{U}(T)$.

REMARK 5.8. Let $X$ be an infinite-dimensional complex Hilbert space, and let $T \in L(X)$ be the operator defined in Example 5.4. Since $T$ is quasinilpotent and $\mathcal{R}\left(T^{n}\right)$ is closed for every $n \in \mathbb{N}$, from Proposition 5.7 it follows that $\mathcal{R}(f(T))$ is closed for every $f \in \mathcal{U}(T)$ (that is, for every complex-valued function $f$ which is holomorphic in an open neighborhood of zero). In addition, we remark that the hypotheses of Proposition 5.7 are also satisfied by the operator constructed in the proof of [Ap, Theorem 3], with spectrum equal to the countable compact subset $\sigma$ of the complex plane.

Note added in proof. About at the same time of the revised version of our article, W. R. Wogen wrote a paper entitled "Algebras of operators and closed range" (to appear in Proc. Amer. Math. Soc.), in which a result similar to Theorem 3 of [Ap] is obtained for a nonempty finite subset $\sigma$ of $\mathbb{C}$, ensuring nonalgebraicity of the operator $T$ with $\sigma(T)=\sigma$, and closedness of the ranges of all operators in the norm closure of the polynomials in $T$. Clearly, for $\sigma=\{0\}$, this provides a further example of a nonnilpotent quasinilpotent operator all the iterates of which have closed range.

\section{References}

[Al] G. R. Allan, Sums of idempotents and a lemma of N. J. Kalton, Studia Math. 121 (1996), 185-192.

[Ap] C. Apostol, On the closed range points in the spectrum of operators, Rev. Roumaine Math. Pures Appl. 21 (1976), 971-975. 
[At] A. Atzmon, Operators which are annihilated by analytic functions and invariant subspaces, Acta Math. 144 (1980), 27-63.

[AD] B. Aupetit and D. Drissi, Some spectral inequalities involving generalized scalar operators, Studia Math. 109 (1994), 51-66.

[B1] L. Burlando, On the iterates of a paracomplete operator, J. Operator Theory 36 (1996), 357-377.

[B2] - A generalization of the uniform ergodic theorem to poles of arbitrary order, Studia Math. 122 (1997), 75-98.

[B3] - Generalizations of Cesàro means and poles of the resolvent, ibid. 164 (2004), $257-281$.

[D1] D. Drissi, On a theorem of Gelfand and its local generalizations, ibid. 123 (1997), 185-194.

[D2] - On a generalization of Lumer-Phillips' theorem for dissipative operators in a Banach space, ibid. 130 (1998), 1-7.

[DZ] D. Drissi and J. Zemánek, Gelfand-Hille theorems for Cesàro means, Quaestiones Math. 23 (2000), 375-381.

[EZ] J. Esterle and M. Zarrabi, Local properties of powers of operators, Arch. Math. (Basel) 65 (1995), 53-60.

[Ge1] I. Gelfand, Ideale und primäre Ideale in normierten Ringen, Mat. Sb. 9 (1941), $41-48$.

[Ge2] - Zur Theorie der Charaktere der Abelschen topologischen Gruppen, Mat. Sb. 9 (1941), 49-50.

[Go] M. Gonzalez, Null spaces and ranges of polynomials of operators, Publ. Mat. 32 (1988), 167-170.

[GO1] M. Gonzalez and V. M. Onieva, On the meromorphic and Schechter-Shapiro operational calculus, J. Math. Anal. Appl. 116 (1986), 363-377.

[GO2] - - - On functions of relatively regular and normally solvable operators, ibid. 133 (1988), 209-213.

[Gr1] S. Grabiner, Ranges of products of operators, Canad. J. Math. 26 (1974), 14301441.

[Gr2] -, Uniform ascent and descent of bounded operators, J. Math. Soc. Japan 34 (1982), 317-337.

[GZ] S. Grabiner and J. Zemánek, Ascent, descent, and ergodic properties of linear operators, J. Operator Theory 48 (2002), 69-81.

$[\mathrm{H}] \quad$ E. Hille, On the theory of characters of groups and semigroups in normed vector rings, Proc. Nat. Acad. Sci. U.S.A. 30 (1944), 58-60.

[HP] E. Hille and R. S. Phillips, Functional Analysis and Semi-Groups, Amer. Math. Soc., 1957.

[K] T. Kato, Perturbation Theory for Linear Operators, Springer, 1966.

$[\mathrm{LM}] \quad$ K. B. Laursen and M. Mbekhta, Operators with finite chain length and the ergodic theorem, Proc. Amer. Math. Soc. 123 (1995), 3443-3448.

[L] D. C. Lay, Spectral analysis using ascent, descent, nullity and defect, Math. Ann. 184 (1970), 197-214.

[MO] M. Mbekhta et O. Ouahab, Opérateur s-régulier dans un espace de Banach et théorie spectrale, Acta Sci. Math. (Szeged) 59 (1994), 525-543.

[MZ] M. Mbekhta et J. Zemánek, Sur le théorème ergodique uniforme et le spectre, C. R. Acad. Sci. Paris Sér. I Math. 317 (1993), 1155-1158.

[Na] M. A. Naimark, Normed Rings, Noordhoff, 1959.

[Ne] O. Nevanlinna, Convergence of Iterations for Linear Equations, Birkhäuser, 1993.

[Sc] H. H. Schaefer, Topological Vector Spaces, Macmillan, 1966. 
[St] M. H. Stone, On a theorem of Pólya, J. Indian Math. Soc. 12 (1948), 1-7.

[TL] A. E. Taylor and D. C. Lay, Introduction to Functional Analysis, 2nd ed., Wiley, 1980 .

[W] H. D. Wacker, Über die Verallgemeinerung eines Ergodensatzes von Dunford, Arch Math. (Basel) 44 (1985), 539-546.

[Y] T. Yamamoto, A note on the spectral mapping theorem, SIAM J. Math. Anal. 2 (1971), 49-51.

[Za] M. Zarrabi, Spectral synthesis and applications to $C_{0}$-groups, J. Austral. Math. Soc. Ser. A 60 (1996), 128-142.

[Ze] J. Zemánek, On the Gelfand-Hille theorems, in: Functional Analysis and Operator Theory, J. Zemánek (ed.), Banach Center Publ. 30, Inst. Math., Polish Acad. Sci., Warszawa, 1994, 369-385.

Dipartimento di Matematica dell'Università di Genova

Via Dodecaneso 35

16146 Genova, Italy

E-mail: burlando@dima.unige.it

Received August 18, 2003

Revised version April 2, 2004 